\title{
$\begin{array}{ll}\text { Research Square } & \begin{array}{l}\text { Preprints are preliminary reports that have not undergone peer review. } \\ \text { They should not be considered conclusive, used to inform clinical practice, } \\ \text { or referenced by the media as validated information. }\end{array}\end{array}$
}

\section{Maternal High Fat Diet and Diabetes Disrupts Transcriptomic Pathways that Regulate Cardiac Metabolism and Cell Fate in Newborn Rat Hearts}

Claudia C. Preston

Sanford Health https://orcid.org/0000-0002-8067-6785

Tricia D. Larsen

Sanford Health

Julie A. Eclov

University of Nebraska Medical Center

Eli J. Louwagie

Sanford Health

Tyler C. T. Gandy

Sanford Health

Randolph S. Faustino

Sanford Health

Michelle L. Baack ( $\square$ Michelle.Baack@SanfordHealth.org )

https://orcid.org/0000-0001-5966-669X

Original investigation

Keywords: PI3K/AKT pathway, mitoribosomes, mitochondrial biogenesis, cardiovascular disease, maternal diabetes and high-fat diet

Posted Date: May 29th, 2020

DOI: https://doi.org/10.21203/rs.3.rs-30050/v1

License: (c) (1) This work is licensed under a Creative Commons Attribution 4.0 International License. Read Full License 


\section{Abstract \\ Background}

Children born to diabetic or obese mothers have a higher risk of heart disease at birth and later in life. Our previous work using chromatin immunoprecipitation sequencing revealed that late-gestation diabetes in combination with maternal high fat (HF) diet cause a distinct fuel-mediated epigenetic reprogramming of rat cardiac tissue during fetal cardiogenesis. The objective of the present study was to investigate the overall transcriptional signature of newborn offspring exposed to the combination of maternal diabetes and maternal HF diet.

\section{Methods}

Gene expression profiling from hearts of diabetes exposed, HF diet exposed, combination exposed and control newborn rats was compared for differential transcriptome expression. Functional annotation, pathway and network analysis was performed on statistically significant differentially expressed genes from the combination exposed group compared with controls. Downstream metabolic assessments included measurement of total and phosphorylated AKT2 and GSK3 $\beta$ assays, as well as quantification of glycolytic capacity by extracellular flux analysis and glycogen staining.

\section{Results}

Transcriptome analysis of newborn rat hearts showed significant changes in cardiac gene expression following exposure to maternal diabetes or HF diet individually, as well as the combination of diabetes + HF compared with controls. Reactome analyses identified expression changes in two key signaling cascades functionally prioritized in male control and combination exposed offspring hearts. These pathways included downregulation of the fibroblast growth factor (FGF) pathway and concomitant downstream PI3K/AKT activation canonically recognized as a regulator of cell metabolism, growth, and development. In contrast, the second pathway exhibited significant upregulation of mitoribosomal signaling that regulates mitochondrial biogenesis, mitophagy and cell fate. Focused bioinformatic analysis on mitochondrial genes enriched in the combination exposed dataset revealed genes associated with diverse aspects of mitochondrial structure, function, and dynamism. Functional biochemical, metabolic, and histochemical assays supported these transcriptome changes, confirming the essential role of mitochondrial energetics in facilitating diabetes- and diet-induced cardiac transcriptome remodeling and phenotype in offspring.

\section{Conclusions}

This study provides the first data accounting for the compounding effects of maternal hyperglycemia and hyperlipidemia on the developmental cardiac transcriptome, and elucidates nuanced and novel features of maternal diabetes and diet on intergenerational regulation of heart health.

\section{Background}

Cardiovascular disease (CVD) is the leading cause of death in the United States and by 2030 is projected to affect $40.5 \%$ of the US population [1]. It is critical to identify high-risk populations and implement targeted prevention in order to decrease this growing burden of disease. The pathogenesis of CVD is influenced over time by both hereditary and environmental factors. Mounting evidence shows that these processes may even begin before birth [2, 3]. Specifically, exposure to excess circulating maternal fuels during critical windows of fetal development increases the lifetime risk of CVD [4-7]. Worldwide, there are annually 21.3 million live births to women with hyperglycemia during pregnancy [8]. Additionally, $35 \%$ of women are obese [9], a co-morbidity that exacerbates this growing problem. Indeed, obese women develop gestational diabetes mellitus (GDM) at odds 4 times higher than non-obese women [10]. For these reasons, finding effective, targeted prevention for this growing and readily identifiable population would significantly lower the burden of heart disease over time.

While it is increasingly recognized that infants born to diabetic or obese mothers have a higher incidence of heart disease at birth and later in life, prevention is significantly hindered because the underlying mechanisms remain unknown. The current standard of care is to optimize maternal blood sugar levels before and during pregnancy [11]. While this has certainly decreased some perinatal morbidities, infants born to mothers with good glycemic control continue to have a higher risk of heart disease [12-15] and gestational diabetes can predispose infants to macrosomia and programmed cardiometabolic disease as adults, even if their mother was treated during pregnancy [16-24]. This suggests there are additional under-recognized, targetable risk factors including lipids. Both maternal diabetes and high fat (HF) diet increase circulating lipids above the normal physiologic hyperlipidemia of pregnancy [25]. We developed a rat model to determine the individual and compounding effects of maternal diabetes and HF diet on cardiac outcomes in offspring. We found that a triad of maternal hyperglycemia, hyperlipidemia and fetal hyperinsulinemia led to progressively worsening mitochondrial dysfunction, impaired cellular bioenergetics, and poorer cardiac function in newborn offspring hearts [26, 27].

We hypothesized that exposure to excess circulating fuels disrupts the in utero gene-environment interaction to program heart disease in the developing fetus, specifically through metabolic and mitochondrial mediated mechanisms. Our previous data using a well-characterized rat model and chromatin immunoprecipitation sequencing (ChIP-Seq) showed that maternal HF diet, especially alongside late-gestation hyperglycemia, caused distinct fuel-mediated epigenetic programming of cardiac metabolism during fetal cardiogenesis [28]. The present study used a cardiac systems biology approach that uncovered specific mechanisms underlying cardiometabolic pathology that may serve as potential targets for personalized therapies. 


\section{Methods}

All experimental methods were carried out in agreement with applicable international, national, and institutional guidelines for the care and use of animals (Animal Welfare Act and National Institutes of Health policies) and were approved by the Sanford Research Institutional Animal Care and Use Committee. Sprague Dawley rats (Harlan Laboratories, Indianapolis, IN) were used in all experiments and housed in Sanford Research's Animal Resource Center, a climatecontrolled, light-dark cycled facility.

Animal model characteristics

Methods and model characteristics of the four animal groups used in this study have been detailed previously [29, 30]. Briefly, young adult female rats received either control or HF diet (Teklad, Harlan Laboratories, Madison, WI) for at least 28 days before mating and throughout pregnancy. Gestational day zero (GD0) was determined by a positive vaginal swab for spermatozoa. On GD14, after confirmation of pregnancy through ultrasound, dams received either citrate buffer $(0.09 \mathrm{M})$ or $65 \mathrm{mg} / \mathrm{kg}$ of intraperitoneal streptozotocin (Sigma Life Sciences, St. Louis, MO) to induce diabetes in the last third of pregnancy. Hyperglycemia was partially controlled with twice daily insulin treatments to keep glucose levels in a target range of 200-400 mg/dl. Dams that received streptozotocin but did not manifest a fasting blood glucose level $\geq 200 \mathrm{mg} / \mathrm{dl}$ were excluded from the study. Delivery ( GD22) yielded postnatal day one (P1) offspring from four distinct groups: exposed to maternal diabetes alone, exposed to maternal HF diet alone, exposed to the combination of both maternal diabetes and HF diet, and control group (Figure 1A). We used all four groups for gene expression analysis with further experiments streamlined to control and combination exposed groups.

Total RNA isolation and quantification

Hearts were extracted from the following four experimental groups of newborn P1 rats: control, diabetes exposed, HF diet exposed, and combination exposed. Immediately after harvesting, samples were snap frozen in liquid nitrogen, and stored at $-80^{\circ} \mathrm{C}$ until RNA extraction. Each experimental group consisted of a pool of male and female rat hearts. Total RNA was extracted from the whole heart with TRIzol and purified using an affinity resin column (Qiagen RNeasy Mini kit, Germantown, MD) according to manufacturer's protocol. Total RNA concentration was performed using spectrophotometric analysis measurement (absemission A260/A280) using NanoDrop 2000 UV-Vis Spectrophotometer (Thermo Fisher Scientific Inc. Waltham, MA). RNA sample integrity was assessed by electropherogram analysis on an Agilent 2100 Bioanalyzer (Agilent Technologies, Santa Clara, CA) and only samples with RNA integrity number (RIN) scores > 8 were used for microarray labeling and hybridization.

Microarray hybridization and data analysis

Microarray hybridization was performed by the Analytical Genomics Core Facility (Sanford Burnham Medical Discovery Institute, Lake Nona, Orlando, FL) using GeneChip Rat Gene 1.0 ST arrays (Affymetrix, Santa Clara, CA) according to manufacturer's protocol. Briefly, total isolated RNA (100 ng) from each sample was converted to cDNA utilizing SuperScript III First Strand Synthesis Supermix (Invitrogen, Life Technologies Corporation, Carlsbad, CA). Labeled complimentary RNA (cRNA), synthesized and amplified from the double-stranded cDNA template, was fragmented and hybridized onto GeneChip arrays. As a measure of quality control of the fragmented biotin-labeled cRNA, a prior hybridization of a test-3 array was performed and analyzed. GeneChip 3000 scanner (Affymetrix, Santa Clara, CA) was used to scan and quantitatively analyze images of hybridized GeneChip arrays. Intensity values for each probe cell in the arrays were calculated by GeneChip software.

Data normalization and analysis was performed using GeneSpring GX 14.01 (Agilent Technologies, Palo Alto, CA). Probe cell intensities were used to calculate an average intensity for each set of probe pairs representing a gene. Quality control (QC) filtering was performed on the normalized intensity values and entities were clustered into four conditions: diabetes, HF diet, combination exposed and controls. Gene expression profiles for each condition were visualized as volcano plots to identify genes significantly upregulated or downregulated in each group.

Bioinformatics analysis

\section{Gene ontology deconvolution and functional annotation analysis}

Statistically significant gene expression profiles from each comparison were separated into upregulated and downregulated lists for functional annotation, Kyoto Encyclopedia of Genes and Genomes (KEGG), and Reactome pathway enrichment analysis using the Database for Annotation, Visualization and Integrated Discovery (DAVID) Bioinformatics Resources v6.8 (https://david.ncifcrf.gov/). To determine over representation or enrichment, the DAVID algorithm employs a modified Fisher's exact test that is incorporated into a score that reports relative priority. Distinct gene lists for each comparison were submitted to DAVID using Entrez Gene identifiers for functional annotation analysis. A high classification stringency was selected to maintain robust groups, and scores were reported for KEGG and Reactome pathways when applicable. Further functional pathway analysis of a given gene list was done through Reactome pathways database analysis tool (Reactome v69; https://reactome.org/).

\section{Functional networks analysis and gene targets prioritization}

Ingenuity pathway analysis (IPA; Qiagen, Germantown, MD) was performed to map functional gene networks defined by the quality-filtered transcriptome. Highest priority network scores were determined and all the gene relationships, i.e. functional interactions among genes, were exported from IPA for use in Cytoscape v3.7.1 (https://cytoscape.org/) for further network analysis. Prioritization of gene targets was achieved through graph theory analysis tools within Cytoscape. Molecule Activity Predictor Analysis module in IPA was used to predict activation or inhibition of non-focused neighboring molecules, defined by IPA as molecules not included in the uploaded list of genes/molecules, within the functional network. This prediction analysis is based on the expression of the focused molecules, also known as statistically significant genes, within the network and predicts either upstream and/or downstream activities.

Page 3/21 
MitoCarta 2.0 database (Broad Institute, Cambridge, MA) was used to determine the mitochondrial-associated genes in our list of statistically significant genes. MitoCarta 2.0 is an online repository of 1158 mammalian (human and mouse) genes encoding proteins where their mitochondrial localization has been validated by various methods. We cross-referenced data from MitoCarta with data from the current study to identify mitochondrial-related genes in our gene expression dataset. The recently updated mouse MitoCarta 2.0 database used in the present analysis can be found at the following website: (https://www.broadinstitute.org/files/shared/metabolism/mitocarta/mouse.mitocarta.2.0.html).

\section{Quantitative RT PCR}

RNA was extracted from newborn (P1) rat hearts using the RNeasy Fibrous Tissue Mini kit (Qiagen, Germantown, MD) following manufacturer's protocol. RNA integrity was assessed by electropherograms using 2100 BioAnalyzer (Agilent Technologies, Santa Clara, CA) and demonstrated RIN scores of 9.2-10 (average=9.8). RNA concentration from two groups, control and combination exposed, was measured by Epoch spectrophotometer (BioTek, Winooski, VT). Complementary DNA (cDNA) was synthesized using iScript cDNA Synthesis Kit and T100 Thermal Cycler (Bio-Rad, Hercules, California). Quantitative PCR (qPCR) was performed by TaqMan Gene Expression Assays approach with ABsolute Blue QPCR Mix (Thermo Fisher Scientific, Waltham, MA) using an ABI7500 qPCR system (Thermo Fisher Scientific, Waltham, MA). Beta-2-microglobulin $(B 2 m)$ was used as the reference gene. Cardiac expression relative to $B 2 m$ was compared between the control and combination exposed groups ( $\mathrm{n}=6 \mathrm{males} /$ group). B2m, mitochondrial ribosomal protein L19 (Mrp/19), mitochondrial ribosomal protein S27 (Mrps27), peroxisome proliferator-activated receptor gamma coactivator 1 alpha (Ppargc1a) and fibroblast growth factor receptor 2 (Fgfr2) probe/primer sets were obtained from Thermo Fisher Scientific (Waltham, MA), and death associated protein 3 (Dap3) probe/primer set was obtained from Integrated DNA Technologies (Coralville, IA).

Western blot analysis

Newborn (P1) rat hearts from control and combination exposed males were homogenized and sonicated in RIPA buffer (50 mM Tris (pH 7.5$)$, 150 mM NaCl, $1 \%$ Triton X, 0.5\% deoxycholate, $0.1 \%$ sodium dodecyl sulfate) with cOmplete protease inhibitor cocktail (Roche, Indianapolis, IN) and phosphatase inhibitor cocktail (Sigma-Aldrich, St. Louis, MO). Protein concentrations were measured using the DC Protein Assay kit (Bio-Rad, Hercules, CA) and Cytation 3 Spectrophotometer (BioTek, Winooski, VT). Protein $(20 \mu \mathrm{g})$ was prepared using Laemmli buffer and reducing agent then subjected to electrophoresis on 4-15\% Criterion TGX Gels using Tris/Glycine/SDS buffer (Bio-Rad). MagicMark XP Western Protein Standard (Thermo Fisher Scientific, Waltham, MA) was used to identify band size. Gels were transferred to PVDF membranes using Trans-Blot Turbo Transfer System (Bio-Rad). Membranes were dried, rehydrated in methanol, washed in TBS, blocked in TBS containing $10 \%$ Clear Milk Blocking Buffer (Thermo Fisher Scientific) and then incubated overnight at $4^{\circ} \mathrm{C}$ with primary antibody. After washing in TBS-T, membranes were blocked again and incubated with secondary antibody for $1 \mathrm{hr}$, using goat anti-rabbit IgG-HRP for reference proteins (Southern Biotech, Birmingham, AL) or donkey anti-rabbit IgG IRDye 680RD (LI-COR, Lincoln, NE) for proteins of interest. HRP exposed bands were visualized using Luminata Forte HRP Chemiluminescence Substrate (Thermo Fisher Scientific). Images were captured using a ChemiDoc MP Imaging System (Bio-Rad) and densitometric analysis was done using ImageJ. Optical density (OD) measurements from tested proteins were normalized to primary reference protein $\beta$-ACTIN. Voltage-dependent anion channel or porin (VDAC) and translocase of outer mitochondrial membrane 20 (TOMM20), both outer mitochondrial membrane proteins, were used as secondary references.

Enzymatic assays

Insulin and c-peptide levels were measured on 25ul of newborn (P1) serum using the MILLIPLEX Map Rat Metabolic panel (MilliporeSigma, Burlington, MA) as previously done $[26,29]$ and Total Protein kinase B isoform 2 (AKT2) and Ser473-phosphorylated AKT2 (regulatory site for insulin signaling) were measured using the MILLIPLEX Map Phospho/Total AKT2 2-plex Magnetic Bead Panel (MilliporeSigma, Burlington, MA) according to manufacturer's protocol as described [29]. Briefly, 15ug of aforementioned newborn (P1) rat heart protein was incubated overnight with antibody coated beads. The beads were then washed and incubated with Detection Antibody for $1 \mathrm{hr}$. Streptavidin-PE was then used as a reporter molecule. The plates were read and analyzed using Luminex 200 Milliplex Analyzer (MilliporeSigma, Burlington, MA).

Total glycogen synthase kinase 3 beta (GSK3 $\beta$ ) and Ser9 phosphorylated GSK3 $\beta$ were measured from aforementioned newborn (P1) rat heart protein using GSK3 $\beta$ (Total/Phospho) Multispecies InstantOne ELISA Kit (Thermo Fisher Scientific, Waltham, MA) according to manufacturer's protocol. In short, 30 mg of protein lysate was added to the ELISA plate and incubated with Antibody Cocktail for $1 \mathrm{hr}$. The wells were then washed with Wash Buffer and exposed to Detection Reagent. After $15 \mathrm{~min}$, Stop Solution was added and the plate was read at $450 \mathrm{~nm}$ using Cytation 3 Spectrophotometer (BioTek, Winooski, VT).

Extracellular Flux (XF) analyses

A glycolytic stress test (GST) was used to compare basal and maximal glycolysis and reserve capacity of primary isolated neonatal (P1) rat cardiomyocytes (NRCM) from control and combination exposed offspring on Seahorse XF24 analyzer (Agilent Technologies, Palo Alto, CA). Methods and experimental validation were similar to that previously described [26]. Briefly, newborn hearts were harvested in ice-cold HBSS. Atria were removed and ventricles were minced then digested with $2 \mathrm{mg} / \mathrm{mL}$ DNase I and 0.15\% Trypsin. Digestion was stopped with bovine serum (BS) and cells were pelleted and transferred to 10:1 DMEM-1 (DMEM with $10 \% \mathrm{BS}$ and $1 \%$ penicillin/streptomycin) with DNase I. Cells were incubated on an uncoated plastic dish for $1 \mathrm{hr}$ at $37^{\circ} \mathrm{C}$ in $5 \% \mathrm{CO}_{2}$ to remove rapidly adhering fibroblasts. Live NRCM were then counted and plated at a seeding density of 40,000 cells/well to $0.1 \%$ gelatin coated V7-PS 24 -well microplates in DMEM-2 (DMEM-1 with $100 \mu \mathrm{M}$ bromodeoxyuridine) and incubated overnight $\left(12 \mathrm{hr}\right.$ ) at $37^{\circ} \mathrm{C}$ with humidified $5 \% \mathrm{CO}_{2}$. The following morning, media was changed to XF base media (Agilent Technologies), incubated without $\mathrm{CO}_{2}$ for $1 \mathrm{hr}$, and then oxygen consumption rates (OCR) and extracellular acidification rates (ECAR) were measured at baseline and following injections to yield final well concentrations. Conditions were as follows:

- Port A: 10 mM D-(+)-glucose (Sigma G8644) 
- Port B: $2 \mu \mathrm{M}$ rotenone (Sigma R8875) + $4 \mu \mathrm{M}$ antimycin A (Sigma A8674), respiratory complex inhibitors used to drive anaerobic glycolysis

- Port C: $200 \mu \mathrm{M}$ monensin (Sigma M5273) + $0.25 \mu \mathrm{M}$ carbonyl cyanide-4-(trifluoromethoxy) phenylhydrazone (FCCP, Sigma C2920) which uncouples aerobic respiration to ensure attainment of maximal anaerobic glycolysis

Outcome measures included: baseline glycolysis, glycolysis following glucose injection, and maximal glycolysis (defined as peak ECAR following rotenone/antimycin) because FCCP/monensin did not usually increase ECAR further. The proton production rate (PPR) was calculated as originally described by Mookerjee et al. to discern acidification from anaerobic glycolysis vs mitochondrial respiration [26, 31].

Histopathology

Formalin fixed and paraffin embedded P1 rat hearts $(n=13)$ were sectioned and stained with periodic acid-Schiff (PAS) stain to qualitatively compare glycogen stores. In brief, biventricular cross-sections were deparaffinized and rehydrated, stained with $0.5 \%$ periodic acid for 5 minutes then Schiff solution for 15 minutes and washed in running water 10 for minutes. Sections were then counterstained with hematoxylin, dehydrated, and mounted with a coverslip. Sections were digitally imaged with the Aperio VERSA 8 automated slide scanner and qualitatively analyzed using the Aperio Image Scope Software (Leica Biosystems Imaging, Buffalo Grove, IL).

Mitochondrial copy number

Total DNA was extracted from P1 whole hearts ( $n=6-7$ males/group) via DNeasy Blood and Tissue Kit (Qiagen, Germantown, MD) following manufacturer's instructions. DNA integrity and concentrations were determined using Epoch spectrophotometer (BioTek, Winooski, VT). Relative mitochondrial DNA copy number was determined using qPCR with primers designed for mitochondrial control region (D-loop; Integrated DNA Technologies, Coralville, IA) and cytochrome- $c$ oxidase I (Mt-co1; Thermo Fisher Scientific, Waltham, MA) as previously described [26, 32]. All qPCR reactions were run in triplicate in ABsolute Blue QPCR Mix (Thermo Fisher Scientific) on a Stratagene Mx3000P thermocycler (Agilent Technologies, Santa Clara, CA). Gene-specific standard curves were calculated using rat mitochondrial DNA and MxPro software (Agilent Technologies, Santa Clara, CA) and used to calculate relative mitochondrial DNA copy number.

Statistical analysis and data availability

Statistical analysis of the microarray gene expression was performed using the unpaired unequal variance $t$-test (Welch test). The hierarchical clustering for groups and entities was performed using Euclidean distance metric and Ward's linkage algorithm. Statistical significance was set at fold change (FC) $>1.25$ and $p$-value $(p)<0.05$. The microarray datasets generated and analyzed in this study are available in the NCBI Gene Expression Omnibus (GEO) database under accession number GSE150649. Results from PCR, protein expression, mitochondrial copy number and extracellular flux analyses were analyzed using student's T-test or Mann-Whitney to detect significant differences between P1 male controls vs combination exposed offspring. These analyses were done using Prism software (GraphPad Software, La Jolla, CA) and statistical significance was set at $\mathrm{p}<0.05$.

\section{Results}

\section{Maternal diabetes and high-fat diet alter the transcriptome of the newborn offspring heart}

Newborn rat hearts were examined by microarray analysis to determine transcriptome remodeling prompted by in utero exposures. Four groups were defined: controls $(n=7)$, diabetes exposed $(n=6)$, HF diet exposed $(n=5)$ and combination exposed $(n=5 ;$ Fig. 1A). Principal component analysis and class prediction modeling (Figs. 1B and C) confirmed reproducibility and consistency of transcriptional profiles. To characterize differentially expressed genes in this study, exposure groups were compared in a pairwise fashion to controls as well as to other exposure groups (Additional File 1: Figure S1, and Additional File 2: Tables S1-6). Of the controls vs exposure pairs analyzed, the greatest number of significant transcriptomic changes was detected for the combination vs control comparison with 323 differentially expressed genes (DEG; Additional File 1: Figure S1A, right panel). Diabetes or HF diet alone versus controls did not return a large enough number of differentially expressed genes to identify significant functional enrichment categories (Additional File 1: Figure S1A, left and middle panel). Analysis of non-control pairs identified the combination exposed group vs HF diet comparison as having the largest number of differentially expressed genes (Additional File 1: Figure S1B). The comparisons of combination exposed vs diabetes and diabetes vs HF diet elicited 245 and 60 DEGs respectively (Additional File 1: Figure S1B). Moving forward, we prioritized the combination vs control comparison for bioinformatic enrichment analysis as the synergistic effects of diabetes and HF diet on transcriptome remodeling, shown by the large number of significantly changed genes, is highly amenable to translational application.

\section{Cardiometabolic pathways are prioritized in expression profiling of offspring hearts exposed to maternal high-fat diet and diabetes}

DAVID functional annotation analysis of the upregulated gene list from the combination vs control comparison identified 'Mitochondrial translation' (elongation and termination), 'Ribosomal protein' and 'Ribonucleoprotein' as functional category terms within the most prioritized cluster (Cluster 1 , enrichment score: 2.112; Table 1). Cys2-His2 (C2H2) zinc finger was the prioritized functional term in the downregulated DEGs (Cluster 1, enrichment score: 1.329; Table 1). Further enrichment analysis of up and downregulated gene lists using the Reactome Pathway Database prioritized 'Metabolism of proteins' within the upregulated list of genes from the combination exposed vs control comparison, with identification of 'Mitochondrial translation', 'Mitochondrial translation termination', 'Mitochondrial translation elongation' and 'Mitochondrial translation initiation' terms (Fig. 2A). Genes associated with this prioritized functional class included the following mitoribosomal genes: mitochondrial ribosomal protein L38 (Mrp/38), mitochondrial ribosomal protein S10 (Mrps10), Mrp/19, Mrps27, mitochondrial ribosomal protein L40 (Mrp/40), in addition to Dap3 (also known as Mrps29), a regulator of mitochondrial dynamism and cell fate [3335]. Moreover, analysis of the downregulated gene set prioritized 'Disease of signal transduction' (Fig. 2B). Functional terms identified with this pathway

Page 5/21 
included 'Fibroblast growth factor receptor (FGFR) signaling' associated terms, including 'Phosphoinositide 3-kinase (PI3K) cascade', with the following genes enriched: fibroblast growth factor 7 (Fgf7), Fgfr2, and RNA polymerase II subunit A (Polr2a; Fig. 2B). 
Table 1

Functional pathway enrichment analysis of significantly upregulated and downregulated genes in Combination exposed vs Control group. The enrichment an on differentially exposed genes (DEG) was performed with DAVID Functional annotation software using a high stringency. Depicted here for the upregulated DI two distinctive annotation clusters (enrichment score shown in parenthesis) and six clusters for the downregulated list, with their respective functional terms, $r$

\begin{tabular}{|c|c|c|c|c|c|c|c|c|c|}
\hline Upregulated & & Category & Term & Count & $\begin{array}{l}\mathrm{p}- \\
\text { value }\end{array}$ & $\begin{array}{l}\text { Fold } \\
\text { Enrichment }\end{array}$ & Bonferroni & Benjamini & FD \\
\hline & $\begin{array}{l}\text { Annotation } \\
\text { Cluster } 1\end{array}$ & REACTOME_PATHWAY & $\begin{array}{l}\text { R-RNO-5389840: } \\
\text { Mitochondrial translation } \\
\text { elongation }\end{array}$ & 6 & 0.0006 & 8.680 & 0.054 & 0.054 & $0 . \epsilon$ \\
\hline & $(2.112)$ & REACTOME_PATHWAY & $\begin{array}{l}\text { R-RNO-5419276: } \\
\text { Mitochondrial translation } \\
\text { termination }\end{array}$ & 6 & 0.0006 & 8.483 & 0.060 & 0.031 & 0.6 \\
\hline & & UP_KEYWORDS & Ribosomal protein & 5 & 0.0754 & 3.124 & 1.000 & 0.642 & 60 \\
\hline & & UP_KEYWORDS & Ribonucleoprotein & 5 & 0.1382 & 2.499 & 1.000 & 0.738 & 82 \\
\hline & $\begin{array}{l}\text { Annotation } \\
\text { Cluster } 2\end{array}$ & GOTERM_CC_DIRECT & $\begin{array}{l}\text { GO:0016021, integral } \\
\text { component of membrane }\end{array}$ & 19 & 0.9999 & 0.540 & 1.000 & 1.000 & 10 \\
\hline & $(0.00002)$ & UP_KEYWORDS & Transmembrane helix & 21 & 0.9999 & 0.543 & 1.000 & 1.000 & 10 \\
\hline & & UP_KEYWORDS & Transmembrane & 21 & 1.0000 & 0.541 & 1.000 & 1.000 & 10 \\
\hline & & UP_KEYWORDS & Membrane & 26 & 1.0000 & 0.571 & 1.000 & 1.000 & 10 \\
\hline \multirow[t]{21}{*}{ Downregulated } & & Category & Term & Count & $\begin{array}{l}\mathrm{p}- \\
\text { value }\end{array}$ & $\begin{array}{l}\text { Fold } \\
\text { Enrichment }\end{array}$ & Bonferroni & Benjamini & FD \\
\hline & $\begin{array}{l}\text { Annotation } \\
\text { Cluster } 1\end{array}$ & INTERPRO & $\begin{array}{l}\text { IPR015880:Zinc finger, } \\
\text { C2H2-like }\end{array}$ & 7 & 0.031 & 2.93 & 1.000 & 0.93 & 33 \\
\hline & $(1.329)$ & INTERPRO & IPR007087:Zinc finger, C2H2 & 7 & $\underset{*}{0.040}$ & 2.76 & 1.000 & 0.88 & 41 \\
\hline & & SMART & SM00355:ZnF_C2H2 & 7 & 0.085 & 2.24 & 0.999 & 1.00 & 60 \\
\hline & $\begin{array}{l}\text { Annotation } \\
\text { Cluster } 2\end{array}$ & INTERPRO & $\begin{array}{l}\text { IPR000504:RNA recognition } \\
\text { motif domain }\end{array}$ & 3 & 0.240 & 3.18 & 1.000 & 1.00 & 97 \\
\hline & $(0.518)$ & INTERPRO & $\begin{array}{l}\text { IPR012677:Nucleotide- } \\
\text { binding, alpha-beta plait }\end{array}$ & 3 & 0.306 & 2.66 & 1.000 & 1.00 & 99 \\
\hline & & GOTERM_MF_DIRECT & $\begin{array}{l}\text { GO:0000166 nucleotide } \\
\text { binding }\end{array}$ & 3 & 0.380 & 2.25 & 1.000 & 1.00 & 99 \\
\hline & $\begin{array}{l}\text { Annotation } \\
\text { Cluster } 3\end{array}$ & INTERPRO & $\begin{array}{l}\text { IPR000719:Protein kinase, } \\
\text { catalytic domain }\end{array}$ & 4 & 0.361 & 1.85 & 1.000 & 1.00 & 99 \\
\hline & $(0.327)$ & INTERPRO & $\begin{array}{l}\text { IPR008271:Serine/threonine- } \\
\text { protein kinase, active site }\end{array}$ & 3 & 0.398 & 2.17 & 1.000 & 1.00 & 99 \\
\hline & & INTERPRO & $\begin{array}{l}\text { IPR011009:Protein kinase- } \\
\text { like domain }\end{array}$ & 4 & 0.409 & 1.71 & 1.000 & 1.00 & 99 \\
\hline & & INTERPRO & $\begin{array}{l}\text { IPR017441:Protein kinase, } \\
\text { ATP binding site }\end{array}$ & 3 & 0.485 & 1.83 & 1.000 & 1.00 & 99 \\
\hline & & UP_KEYWORDS & Kinase & 4 & 0.486 & 1.52 & 1.000 & 0.99 & 99 \\
\hline & & GOTERM_MF_DIRECT & $\begin{array}{l}\text { GO:0006468 protein } \\
\text { phosphorylation }\end{array}$ & 3 & 0.598 & 1.48 & 1.000 & 1.00 & 10 \\
\hline & & SMART & SM00220:S_TKc & 3 & 0.623 & 1.41 & 1.000 & 1.00 & 10 \\
\hline & $\begin{array}{l}\text { Annotation } \\
\text { Cluster } 4\end{array}$ & INTERPRO & $\begin{array}{l}\text { IPR007110:Immunoglobulin- } \\
\text { like domain }\end{array}$ & 4 & 0.427 & 1.66 & 1.000 & 1.00 & 99 \\
\hline & $(0.279)$ & INTERPRO & $\begin{array}{l}\text { IPR003599:Immunoglobulin } \\
\text { subtype }\end{array}$ & 3 & 0.515 & 1.73 & 1.000 & 1.00 & 99 \\
\hline & & SMART & SM00409:IG & 3 & 0.661 & 1.32 & 1.000 & 1.00 & 10 \\
\hline & $\begin{array}{l}\text { Annotation } \\
\text { Cluster } 5\end{array}$ & UP_KEYWORDS & Membrane & 33 & 0.465 & 1.05 & 1.000 & 1.00 & 99 \\
\hline & $(0.273)$ & UP_KEYWORDS & Transmembrane helix & 28 & 0.490 & 1.05 & 1.000 & 0.99 & 99 \\
\hline & & UP_KEYWORDS & Transmembrane & 28 & 0.500 & 1.04 & 1.000 & 0.99 & 99 \\
\hline & & GOTERM_MF_DIRECT & $\begin{array}{l}\text { GO:0016021 integral } \\
\text { component of membrane }\end{array}$ & 23 & 0.707 & 0.96 & 1.000 & 1.00 & 10 \\
\hline
\end{tabular}




\begin{tabular}{|c|c|c|c|c|c|c|c|c|c|}
\hline Upregulated & & Category & Term & Count & $\begin{array}{l}\mathrm{p}- \\
\text { value }\end{array}$ & $\begin{array}{l}\text { Fold } \\
\text { Enrichment }\end{array}$ & Bonferroni & Benjamini & FD \\
\hline & $\begin{array}{l}\text { Annotation } \\
\text { Cluster } 6\end{array}$ & INTERPRO & $\begin{array}{l}\text { IPR000276:G protein- } \\
\text { coupled receptor, rhodopsin- } \\
\text { like }\end{array}$ & 3 & 0.994 & 0.43 & 1.000 & 1.00 & 10 \\
\hline & $(0.002)$ & INTERPRO & $\begin{array}{l}\text { IPR017452:GPCR, } \\
\text { rhodopsin-like, 7TM }\end{array}$ & 3 & 0.996 & 0.40 & 1.000 & 1.00 & 10 \\
\hline & & UP_KEYWORDS & G-protein coupled receptor & 3 & 0.997 & 0.39 & 1.000 & 1.00 & 10 \\
\hline & & UP_KEYWORDS & Transducer & 3 & 0.997 & 0.38 & 1.000 & 1.00 & 10 \\
\hline
\end{tabular}

Gene regulatory network disruption in developing offspring identifies a functional hub regulating metabolism and cell fate

To investigate gene networks underlying transcriptome remodeling of the combination exposed offspring hearts, IPA was used to map known molecular interactions among genes identified in the present study. All subnetworks that capture gene interactions within the present dataset were integrated into an inclusive, overall network displayed in a circular layout (Fig. 3A). Increased edge density (gray lines) in the lower right quadrant identifies hubs, or highly connected nodes within this network. Focusing on the most prioritized subnetwork with an enrichment score of 39 revealed over representation of 'Cell Cycle', 'Protein Synthesis', and 'Hair and Skin Development/Function' categories (Fig. 3B). Predictive network modeling applied to this subnetwork identified potential Serine/Threonine-protein kinase B ( $A k t)$ inhibition given the net synergistic gene expression changes in the present dataset (Fig. 3B). In addition, this subnetwork integrated up and downregulated genes that represented mitoribosomal signaling and FGF cascades (Fig. 3B). Topology analysis of this prioritized subnetwork identified $A k t$ as a functional hub with the highest betweenness centrality score (Fig. 3C). To identify network hubs regulating informational flow, Akt, Cyclin dependent kinase 1 (Cdk1), and Cyclin A were identified as molecules with the highest closeness centrality measures in the present network (Fig. 3D). Together, these data identifies Akt as a strong candidate pathway involved in reprogramming of diabetes and HF diet exposed cardiac tissue.

\section{Expression Dysregulation Of Mitochondrial Specific Genes}

The overall bioinformatic enrichment data alongside previously published changes in bioenergetics and metabolic functions [26] prompted a focused query on genes regulating mitochondrial function. Intersection of prioritized up and downregulated transcriptional profiles in our study with the independently curated MitoCarta 2.0 database [36] that includes 1158 mitochondrial associated genes, revealed 32 upregulated mitochondrial genes in the combination vs control comparison with no observation of common downregulated genes (Fig. 4A; Additional File 3: Table S7). These 32 genes could be further sub-classified into multiple categories of mitochondrial structure and function, including 'Cell Signaling and Cell Fate', 'Mitoribosomes and Biogenesis', 'Dynamism', 'Oxidative Phosphorylation', 'Metabolism', 'Oxidative Repair' and other mitochondrial functions (Fig. 4B). Candidate genes for validation included Ppargc1a (encoded protein also known as PGC1a, a master regulator of mitochondrial biogenesis), as well as genes that encode mitoribosomal proteins Mrp/19, Mrps27 and Dap3 (Fig. 4C). Of these, validation by qPCR confirmed that Ppargc1a and Mrp/19 mRNA expression were significantly higher in the combination exposed P1 males when compared to controls (FC 2.24 and 2.28 respectively, $\mathrm{p}<0.05, \mathrm{n}=6$ /group; Fig. 4C, top row). Mrps27 and Dap3 mRNA expression was not different between the two groups (Fig. 4C, top row). Immunoblotting to assess relative expression of protein products for each of these genes confirmed higher expression of PGC1a ( $n=4$ /group, $p<0.05)$, non-significant trends towards higher expression for MRPL19 and DAP3, and no observed change for MRPS27 (Fig. 4C, bottom row). In line with this, we previously reported a higher mitochondrial DNA copy number in HF diet and combination exposed offspring hearts [26]. In this study, we did not detect a higher mitochondrial DNA copy number by PCR (Fig. 4D). However, combination exposed hearts had higher expression of outer mitochondrial membrane proteins TOMM20 or VDAC with significant increases observed in the latter compared to controls.

\section{Downregulated FGFR2/PI3K/AKT activation underlies metabolic switching from glycolysis to gluconeogenesis in the combination exposed offspring hearts}

We previously demonstrated that combination exposed offspring (P1) are exposed to high levels of circulating maternal glucose and lipids which incite insulin and C-peptide overproduction in the offspring [26-29, 37]. This triad of maternal hyperglycemia and hyperlipidemia and fetal hyperinsulinemia causes insulin resistance with attendant downregulation of growth hormone receptors and less PI3K/AKT activation to drive a glycolysis-to-gluconeogenesis switch [29, 3840]. Informed by the observed disruption in the gene regulatory network in the present study, circulating insulin, c-peptide, and the cardiac Fgfr2 gene and FGFR2 protein expression were assessed. Insulin and c-peptide levels were significantly higher in combination exposed offspring compared to controls (539 \pm 77 vs $8,103 \pm 2,337 \mathrm{pg} / \mathrm{mL} ; \mathrm{p}=0.005$ and $2,391 \pm 367$ vs $14,449 \pm 4,175 \mathrm{pg} / \mathrm{mL}$ respectively, $\mathrm{p}<0.01 ; \mathrm{n}=10 / \mathrm{group}$ Fig. $5 \mathrm{~A}$ ). Fgfr2 mRNA levels showed a modest decreasing trend in expression (FC -1.93, $p=0.16$ ), with significantly lower FGFR2 protein expression in combination exposed hearts when compared to controls ( $p<0.05$; Fig. 5 A). Examination of total as well as activated (phosphorylated) AKT expression showed no significant differences between control and combination exposed hearts (Fig. 5B), however, AKT phosphorylation changes rapidly in response to fuels, thus levels would be expected to normalize after birth as glucose and insulin levels rapidly decline. GSK3 $\beta$, is regulated by AKT and when activated by phosphorylation, facilitates gluconeogenesis in multiple tissues including the heart $[41,42]$. In the present study, total GSK3 $\beta$ was moderately increased in combination exposed offspring hearts compared to controls $(0.86 \pm 0.28$ vs $0.99 \pm 0.29$, $p<0.05$; Fig. $5 B$, bottom row) with a trend towards higher activation as demonstrated by phosphorylated ( $0.43 \pm 0.21$ vs $0.70 \pm 0.20, p=0.14)$ and a ratio of phosphorylated:total GSK3 $3(0.48 \pm 0.11$ vs $0.73 \pm 0.03, p=0.057)$. (Fig. $5 B$, bottom row). Again, although significance was not reached, cell signaling through phosphorylation is rapid and so downstream metabolism and glycogen storage within the heart is a more accurate measure of longstanding activation due to in utero exposures.

In our previous studies, primary isolated NRCM from diabetes exposed offspring had impaired respiratory and glycolytic capacity [26]. The present study had a smaller male cohort in which cardiomyocytes from combination exposed rat offspring exhibited a similar glycolytic capacity as controls (Fig. 5C, top row). However, PPR calculations showed combination exposed NRCM have impaired aerobic glycolytic capacity and metabolize glucose through anaerobic 
glycolysis instead. Additionally, PPR due to $\mathrm{CO}_{2}$ production in combination exposed cardiomyocytes did not rise with glycolytic stimulation, suggesting impaired glucose uptake which is the rate limiting step of glycolysis (Fig. 5C, bottom row). Moreover, qualitative histological examination by PAS staining revealed increased glycogen deposition in combination exposed offspring hearts (Fig. 5D). Taken together, combination exposed P1 male hearts provide evidence of insulin resistance with a metabolic predilection to switch from glycolysis to gluconeogenesis.

\section{Discussion}

The lifetime risk of CVD remains close to $50 \%$ for men and $38 \%$ for women making it the leading cause of morbidity and mortality in developed countries [43] There is an urgent need to identify targetable triggers of CVD in high-risk populations so that the earliest risk-assessment and personalized interventions can be implemented to decrease this growing burden of disease over time. Infants born to diabetic mothers (IDM) are a high-risk population with $36 \%$ having pathologic ventricular hypertrophy at birth [44] and as young adults having 30\% greater risk of early onset CVD [16]. Developmental consequences follow exposure to excess circulating fuels during critical windows of fetal development [45], specifically for diabetic pregnancy this includes maternal hyperglycemia, hyperlipidemia and fetal hyperinsulinemia. Similarly, these fuels contribute to diabetic cardiomyopathy in adults which has overlapping clinical manifestations with cardiomyopathy found in IDM [46-49]. Unfortunately, even when maternal hyperglycemia is well-controlled during pregnancy, the risk of heart disease in IDM persists [12, 13, 15, 50]; this implicates additional targetable triggers including lipids. We previously used a rat model to show that a maternal HF diet compounded the effects of late-gestation diabetes by exposing the offspring not only to hyperglycemia, but also to hyperlipidemia which increased fetal insulin production, placental lipotoxicity, cardiac dysfunction, and perinatal mortality [26, 51]. The present study used cardiac-specific transcriptomic profiling to identify potential underlying mechanisms. The key finding was identification of distinct, yet overlapping disruptions in glucose-, insulin- and lipid-modulated networks that influence cardiac development and disease (Fig. 6). Specifically, maternal diabetes and HF diet caused down regulation of a well-known glucose and insulin signaling pathway, FGRF2/PI3K/AKT, as well as a concomitant upregulation of fatty acid regulated PGC1a [52]. Both pathways sense nutrient supply to influence cellular metabolism as well as growth, development and aging [39, 40,53].

Cellular metabolism is of critical importance for the heart which must efficiently shift from one metabolic pathway to another in order to maintain efficient contractily under variable energetic demands (aerobic, anaerobic, resting, exercise, starvation, etc). A small decrease in metabolic efficiency can have profound impacts on cardiac function leading to hypertrophy, stiffness, diastolic and systolic dysfunction [54, 55]. Transcriptomic profiling suggests combination exposed offspring hearts have relative insulin resistance with downregulation of growth factor receptors, likely due to in utero fetal hyperinsulinemia which occurs in response to maternal hyperglycemia. Insulin and other growth factors normally activate PI3K to generate 3,4,5 phosphatidylinositol (PIP3) to recruit AKT to the plasma membrane for activation by phosphorylation on the threonine 308 residue (T308) and serine 473 (S473) by phospoinositide-dependent kinase 1 (PDK1) [56]. In the present study, combination exposed P1 offspring had higher insulin and c-peptide levels with concomitant down regulation of FGFR2, but we did not find lower total or S473 phosphorylation of AKT. We speculate that after birth AKT may have been dephosphorylated in the length of time between offspring removal from the in utero diabetic environment and cardiac tissue harvest. Indeed, offspring's glucose and insulin levels fall rapidly after birth and kinetic studies show peak AKT phosphorylation and activity is 15 minutes after insulin receptor activation, and dephosphorylation occurs within 30 minutes [56]. Additionally, AKT activation can be modulated by phosphorylation at unmeasured sites [56]. So while we did not find direct evidence of impaired PI3K/AKT quantity or phosphorylation, we found residual downstream metabolic impairment as evidence this occurred in utero. In line with this, we previously demonstrated that isolated NRCM from combination exposed offspring had lower glycolytic capacity [26]. A limitation of this study is that we did not replicate the same severity of glycolytic impairment, but found impaired aerobic glycolysis, higher GSK3 $\beta$, and more cardiac glycogen deposition, consistent with a chronic shift from glycolysis to gluconeogenesis. This switch carries significant bioenergetic consequences as the newborn heart relies primarily on aerobic glycolysis for ATP production $[57,58]$. Our findings demonstrate that diabetic pregnancy, especially combined with hyperlipidemia precipitates cardiac "insulin resistance" in the developing fetal heart. The transitory nature of the pathway may explain why IDM have diastolic and systolic dysfunction that begins in utero around the time fetal pancreatic endocrine function begins, but then improves after birth [7, 59-61]. Authors propose that when the fetus is no longer exposed to the triad of hyperglycemia, hyperlipidemia and hyperinsulinemia, growth factor receptor expression normalizes, PI3K/AKT activation is no longer suppressed. This postnatal correction, alongside the physiologic transition from glycolysis to fatty acid oxidation during cardiac maturation [57], could explain why cardiomyopathy in IDM improves after birth. However, this does not explain how infants born to diabetic or obese mothers develop early onset CVD in adulthood $[6,16,23,62,63]$.

Both of the pathways we uncovered by transcriptomic profiling are well-known modulators of stem cell fate, which could influence cardiogenesis and aging as it relates to long-term cardiovascular health [64]. The current study adds additional evidence to our previous work, that maternal diabetes and HF diet affect cardiac health through mitochondrial mediated influences on cell fate and aging processes $[26,27,65,66]$. Our lab has shown that primary isolated cardiomyocytes from combination exposed offspring have poorly charged, adynamic, fragmented mitochondria which can produce ROS and incite mitochondrial mediated cell death [26, 27]. Developing cardiomyocytes normally have highly dynamic mitochondria; after birth they undergo rapid mitochondrial biogenesis and ultrastructural differentiation into distinct sub-populations to meet changing metabolic demands [57, 67-69]. Mitochondrial biogenesis requires complex coordination of nuclear and mitochondrial transcription, translation and protein assembly that is regulated by transcriptional regulators including PGC1a [70]. PGC1a is a positive regulator of mitochondrial biogenesis, oxidative phosphorylation, gluconeogenesis, and ROSdetoxification [70], and is regulated at both transcriptional and post-translational levels through similar yet competing PI3K/AKT and MAPK pathways (Fig. 6) [71]. Oxygen, nutrients, and ROS in the heart signal for the insulin-sensitive PI3K/AKT pathway to reduce PGC1a activity; conversely, they signal for MAPK and glucose-responsive AMPK phosphorylation to increase PGC1a transcriptional activity [71]. In this study, newborn offspring exposed to maternal hyperglycemia and hyperlipidemia and fetal hyperinsulinemia demonstrated down-regulated PI3K/AKT and increased PGC1a expression amongst uninhibited MAPK activity. Together, these transciptomic changes would be expected to increase translation (but not transcription) of mitochondrial proteins, mitochondrial biogenesis, and gluconeogenesis while decreasing glycolysis [40]. This shift in metabolic machinery is the resut of coordinated increases in expression of nuclear and mitochondrial encoded genes needed for mitochondrial biogenesis. 
It is critical to point out that while the vast majority of proteins regulating mitochondrial function are encoded by the nuclear genome and transported to the mitochondria, respiratory complex assembly occurs within the mitochondria guided by mitochondrial DNA (mtDNA) transcription and translation[72]. Located within mitochondrial nucleoids, mtDNA coding for the respiratory complex components are translated into proteins by mitochondrial ribosomes or mitoribosomes under the influence of P32 (complement component 1, q subcomponent binding protein or C1qBP; also called gC1qR or HABP1) [72]. All 82 proteins that make up the mitoribosome are encoded by the nuclear genome and transported into mitochondria for assembly [73]. In this study, cardiac transcriptome analyses identified upregulation of mitoribosomal proteins in combination exposed offspring. Specifically upregulated were Mrp/19, Mrp/38, Mrps10, Mrps27, and Dap3, mitoribosomal proteins influenced by P32, an essential RNA-binding factor in mitochondrial translation indispensable for oxidative phosphorylation and embryonic development [74]. Additionally, Mrp/40, a mitoribosomal protein associated with P32, was upregulated in the combination exposed cardiac transcriptome. MRPL40 is located adjacent to the ribosomal polypeptide exit site and is important for synthesis of mitochondrially encoded proteins and their subsequent assembly into oxidative phosphorylation complexes [75].

DAP3 and P32 play additional roles in mitochondrial function and cell fate [33-35, 74]. We previously detailed a sex-specific role for DAP3 in impaired dynamism and mitochondrial quality control in combination exposed male offspring [27]. As a chaperone for mitochondrial protein synthesis, P32 does not affect the amount of mtDNA or mRNA but rather mitochondrial protein homeostasis, respiration [74], and cell survival [76]. This may explain why combination exposed hearts did not have a higher mtDNA copy number but expressed more VDAC, a mitochondrial outer membrane protein that is often used as a surrogate marker of mitochondrial quantity.

Alternatively, expression of genes influencing mitochondrial biogenesis may be upregulated as a compensatory mechanism to increase turnover of dysfunctional mitochondria found in combination exposed NRCM $[26,27]$. Transcriptomic evidence from this study demonstrates a compensatory shift to increase oxidative phosphorylation including up regulation of Ndufc1, Hccs, and Rtn4ip1 which encode proteins necessary for orchestrated assembly of respiratory complexes or their cofactors. Specifically, NDUFC1 is a subunit in mitochondrial Complex I and HCCS is critical for cytochrome c biogenesis which functions in electron transport from Complex III to Complex IV [77, 78]. HCCS is also is a key regulator of cell fate and cardiomyocyte differentiation. Adenylate kinase 2 (Adk2) was also upregulated in combination exposed offspring. Its encoded protein, AK2, is part of the adenylate kinase shuttling pathway, a central metabolic hub which enables the transfer of phosphoryl groups to interconvert 2ADP $\leftrightarrow$ ATP + AMP. This reaction is not only critical for ATP generation but is a sensitive rheostat of cellular energy that regulates the balance of energy storage and utilization through multiple downstream AMP signaling pathways. Specifically, AK2 is highly expressed in cardiac tissue and localized to the mitochondrial intermembrane space where it facilitates conversion of AMP to ADP shuttling. AK2 has an extremely high affinity for AMP which assures that AMP reaching cardiac mitochondria is converted to ADP and channeled into oxidative phosphorylation. During cardiometabolic stress, mitochondrial AK2 activity is increased in response to increased energy demand and the necessity to maintain the cellular adenine nucleotide pool; moreover higher AK2 is often associated with cardiac hypertrophy [79]. Whether or not AK2 is higher in combination than control offspring hearts because mitochondrial number is higher, probably in response to impaired bioenergetics, is unknown.

Identification here of the complex metabolic underpinnings of impaired offspring cardiac development subsequent to the combination of maternal hyperglycemia and hyperlipidemia is a clear and significant advancement in understanding transgenerational cardiopathology in the context of DoHAD. Significantly, the findings of the present study establish a precedent to examine longitudinal changes, as 'transcriptome snapshots' capture single time points and miss nuances of gene expression dynamics. This is crucial for identifying optimal interventional windows during gestation and devising strategies to improve maternal and fetal health.

\section{Conclusions}

Our findings demonstrate that the combination of maternal diabetes and a high-fat diet remodels the transcriptome in developing fetal hearts. These dysregulated gene expression changes are associated with aberrant bioenergetics and cell fate that could modulate risk of cardiac disease early and later in life. Network prioritization of FGF and PI3/AKT signaling cascades, as well as their convergence on PGC1a, revealed the importance of this cluster in facilitating cardiometabolic derangement (Fig. 6). Ultimately, the present findings may also provide a translational advantage by considering maternal hyperlipidemia and the FGF-PI3K/AKT- PGC1a cluster as a targetable hub for prevention and treatment of developmentally programmed heart disease.

\section{Abbreviations}

CVD

Cardiovascular disease

GDM

Gestational diabetes mellitus

HF

High fat

ChIP-SEq

Chromatin immunoprecipitation sequencing

GD

Gestational day

P1

Postnatal day one

RIN

RNA integrity number

QC

Page 10/21 
Quality control

KEGG

Kyoto Encyclopedia of Genes and Genomes

DAVID

Database for Annotation, Visualization and Integrated Discovery

IPA

Ingenuity pathway analysis

CDNA

Complementary DNA

qPCR

Quantitative PCR

B2m

Beta-2-microglobulin

Mrpl19

Mitochondrial ribosomal protein L19

Mrps27

Mitochondrial ribosomal protein

Ppargc1a

Peroxisome proliferator-activated receptor gamma coactivator 1 alpha, gene

Fgfr2

Fibroblast growth factor receptor 2

Dap3

Death associated protein 3

OD

Optical density

VDAC

Voltage-dependent anion channel or porin

TOMM20

Translocase of outer mitochondrial membrane 20

AKT2

Protein kinase B isoform 2

GSK3 $\beta$

Glycogen synthase kinase 3 beta

GST

Glycolytic stress test

NRCM

Neonatal (P1) rat cardiomyocytes

OCR

Oxygen consumption rates

ECAR

Extracellular acidification rates

FCCP

Cyanide-4-(trifluoromethoxy)phenylhydrazone

PPR

Proton production rate

PAS

Periodic acid-Schiff

GEO

Gene expression omnibus

DEG

Differentially expressed gene

$\mathrm{C} 2 \mathrm{H} 2$

Cys2-His2

Mrpl38

Mitochondrial ribosomal protein L38

Mrps10

Mitochondrial ribosomal protein S10

Mrpl40

Mitochondrial ribosomal protein L40

FGFR 
Fibroblast growth factor receptor

PI3K

Phosphoinositide 3-kinase

Fgf7

Fibroblast growth factor 7

Polr2a

RNA polymerase II subunit A

Cdk1

Cyclin dependent kinase 1

PGC1a

Peroxisome proliferator-activated receptor gamma coactivator 1 alpha, protein

IDM

Infants of diabetic mothers

\section{Declarations}

\section{Ethics approval and consent to participate}

All experimental methods involving animals were carried out in agreement with applicable international, national, and institutional guidelines for the care and use of animals (Animal Welfare Act and National Institutes of Health policies) and were approved by the Sanford Research Institutional Animal Care and Use Committee.

\section{Consent for publication}

Not applicable.

\section{Availability of data and materials}

Microarray gene expression datasets used in this manuscript are available in the GEO repository database (https://www.ncbi.nlm.nih.gov/geo/) with number GSE150649. Other data generated or analyzed during this study are included in this published article and its supplementary additional files.

\section{Competing interests}

All authors declare that they have no conflicts of interests, financial or otherwise.

\section{Funding}

The study was financially supported by Sanford Research, Sanford Burnham Collaborative Research Grant (2256538), National Institutes of Health (NIH) NICHD K08HD078504 and NIH Center for Pediatric Research COBRE grant (P20GM103620). Histology support was provided by the Sanford Research Cancer COBRE via support from NIH - NIGMS, P20GM103548.

\section{Authors' contributions}

MLB and RSF are the principal investigators responsible for the work as a whole. MLB conceived and funded the study, completed animal work and RNA extraction. TDL did PCR, protein, serum and tissue analyses. JAE isolated NRCM and completed extracellular flux analyses and EJL assisted in data interpretation. CCP did bioinformatics analysis on transcriptomic data. RSF and CCP did functional annotation and network analyses. CCP drafted the manuscript and figures with creative support from TCTG. All authors assisted in the project as a whole including manuscript overview.

\section{Acknowledgements}

The authors would like to thank the Sanford Animal Research Center (ARC) staff for assistance with animal work.

\section{References}


1. Heidenreich PA, Trogdon JG, Khavjou OA, Butler J, Dracup K, Ezekowitz MD, et al. Forecasting the future of cardiovascular disease in the United States: a policy statement from the American Heart Association. Circulation. 2011;123(8):933-44.

2. Barker DJ. Rise and fall of Western diseases. Nature. 1989;338(6214):371-2.

3. Silveira PP, Portella AK, Goldani MZ, Barbieri MA. Developmental origins of health and disease (DOHaD). Jornal de pediatria. 2007;83(6):494-504.

4. Cho NH, Silverman BL, Rizzo TA, Metzger BE. Correlations between the intrauterine metabolic environment and blood pressure in adolescent offspring of diabetic mothers. J Pediatr. 2000;136(5):587-92.

5. Dong M, Zheng Q, Ford SP, Nathanielsz PW, Ren J. Maternal obesity, lipotoxicity and cardiovascular diseases in offspring. J Mol Cell Cardiol. 2013;55:111-6.

6. Eriksson JG, Sandboge S, Salonen MK, Kajantie E, Osmond C. Long-term consequences of maternal overweight in pregnancy on offspring later health: findings from the Helsinki Birth Cohort Study. Annals of medicine. 2014;46(6):434-8.

7. Ren Y, Zhou Q, Yan Y, Chu C, Gui Y, Li X. Characterization of fetal cardiac structure and function detected by echocardiography in women with normal pregnancy and gestational diabetes mellitus. Prenatal diagnosis. 2011;31(5):459-65.

8. Cho NH, Shaw JE, Karuranga S, Huang Y, da Rocha Fernandes JD, Ohlrogge AW, et al. IDF Diabetes Atlas: Global estimates of diabetes prevalence for 2017 and projections for 2045. Diabetes Res Clin Pract. 2018;138:271-81.

9. Flegal KM, Carroll MD, Ogden CL, Curtin LR. Prevalence and trends in obesity among US adults, 1999-2008. Jama. 2010;303(3):235-41.

10. Najafi F, Hasani J, Izadi N, Hashemi-Nazari SS, Namvar Z, Mohammadi S, et al. The effect of prepregnancy body mass index on the risk of gestational diabetes mellitus: A systematic review and dose-response meta-analysis. Obesity reviews: an official journal of the International Association for the Study of Obesity; 2018.

11. ACOG Practice Bulletin No. 190 Summary: Gestational Diabetes Mellitus. Obstetrics gynecology. 2018;131(2):406-8.

12. Aman J, Hansson U, Ostlund I, Wall K, Persson B. Increased fat mass and cardiac septal hypertrophy in newborn infants of mothers with well-controlled diabetes during pregnancy. Neonatology. 2011;100(2):147-54.

13. Kozak-Barany A, Jokinen E, Kero P, Tuominen J, Ronnemaa T, Valimaki I. Impaired left ventricular diastolic function in newborn infants of mothers with pregestational or gestational diabetes with good glycemic control. Early Hum Dev. 2004;77(1-2):13-22.

14. Xie GH, Zheng Z, Liu TC, Qing LL, Hong XQ, Zha WT, et al. Health care and risk of adverse pregnancy outcomes among diabetic women: an updated metaanalysis. Archives of gynecology and obstetrics. 2019.

15. Ghandi Y, Habibi D, Nasri K, Alinejad S, Taherahmad H, Arjmand Shabestari A, et al. Effect of well-controlled gestational diabetes on left ventricular diastolic dysfunction in neonates. The journal of maternal-fetal \& neonatal medicine: the official journal of the European Association of Perinatal Medicine, the Federation of Asia and Oceania Perinatal Societies, the International Society of Perinatal Obstet. 2018:1-6.

16. Yu Y, Arah OA, Liew Z, Cnattingius S, Olsen J, Sorensen HT, et al. Maternal diabetes during pregnancy and early onset of cardiovascular disease in offspring: population based cohort study with 40 years of follow-up. BMJ. 2019;367:16398.

17. Catalano PM, McIntyre HD, Cruickshank JK, McCance DR, Dyer AR, Metzger BE, et al. The hyperglycemia and adverse pregnancy outcome study: associations of GDM and obesity with pregnancy outcomes. Diabetes Care. 2012;35(4):780-6.

18. Crowther CA, Hiller JE, Moss JR, McPhee AJ, Jeffries WS, Robinson JS. Effect of treatment of gestational diabetes mellitus on pregnancy outcomes. N Engl J Med. 2005;352(24):2477-86.

19. Boney CM, Verma A, Tucker R, Vohr BR. Metabolic syndrome in childhood: association with birth weight, maternal obesity, and gestational diabetes mellitus. Pediatrics. 2005;115(3):e290-6.

20. Bianco ME, Josefson JL. Hyperglycemia During Pregnancy and Long-Term Offspring Outcomes. Curr Diabetes Rep. 2019;19(12):143.

21. Brown J, Martis R, Hughes B, Rowan J, Crowther CA. Oral anti-diabetic pharmacological therapies for the treatment of women with gestational diabetes. Cochrane Database Syst Rev. 2017;1:Cd011967.

22. Bunt JC, Tataranni PA, Salbe AD. Intrauterine exposure to diabetes is a determinant of hemoglobin A(1)c and systolic blood pressure in pima Indian children. J Clin Endocrinol Metab. 2005;90(6):3225-9.

23. Farahvar S, Walfisch A, Sheiner E. Gestational diabetes risk factors and long-term consequences for both mother and offspring: a literature review. Expert review of endocrinology \& metabolism. 2018:1-12.

24. Landon MB, Spong CY, Thom E, Carpenter MW, Ramin SM, Casey B, et al. A multicenter, randomized trial of treatment for mild gestational diabetes. N Engl J Med. 2009;361(14):1339-48.

25. Ryckman KK, Spracklen CN, Smith CJ, Robinson JG, Saftlas AF. Maternal lipid levels during pregnancy and gestational diabetes: a systematic review and meta-analysis. BJOG: an international journal of obstetrics gynaecology. 2015;122(5):643-51.

26. Mdaki KS, Larsen TD, Wachal AL, Schimelpfenig MD, Weaver LJ, Dooyema SD, et al. Maternal high-fat diet impairs cardiac function in offspring of diabetic pregnancy through metabolic stress and mitochondrial dysfunction. American journal of physiology Heart and circulatory physiology. 2016:ajpheart 007952015.

27. Larsen TD, Sabey KH, Knutson AJ, Gandy TCT, Louwagie EJ, Lauterboeck L, et al. Diabetic Pregnancy and Maternal High-Fat Diet Impair Mitochondrial Dynamism in the Developing Fetal Rat Heart by Sex-Specific Mechanisms. International journal of molecular sciences. 2019;20(12).

28. Upadhyaya B, Larsen T, Barwari S, Louwagie EJ, Baack ML, Dey M. Prenatal Exposure to a Maternal High-Fat Diet Affects Histone Modification of Cardiometabolic Genes in Newborn Rats. Nutrients. 2017;9(4). 
29. Baack ML, Forred BJ, Larsen TD, Jensen DN, Wachal AL, Khan MA, et al. Consequences of a maternal high-fat diet and late gestation diabetes on the developing rat lung. PloS one. 2016;11(8):e0160818.

30. Mdaki KS, Larsen TD, Wachal AL, Schimelpfenig MD, Weaver LJ, Dooyema SD, et al. Maternal high-fat diet impairs cardiac function in offspring of diabetic pregnancy through metabolic stress and mitochondrial dysfunction. American journal of physiology Heart circulatory physiology. 2016;310(6):H681-92.

31. Mookerjee SA, Goncalves RL, Gerencser AA, Nicholls DG, Brand MD. The contributions of respiration and glycolysis to extracellular acid production. Biochim Biophys Acta. 2015;1847(2):171-81.

32. Nicholls DG, Darley-Usmar VM, Wu M, Jensen PB, Rogers GW, Ferrick DA. Bioenergetic profile experiment using C2C12 myoblast cells. Journal of visualized experiments: JoVE. 2010(46).

33. Kim HR, Chae HJ, Thomas M, Miyazaki T, Monosov A, Monosov E, et al. Mammalian dap3 is an essential gene required for mitochondrial homeostasis in vivo and contributing to the extrinsic pathway for apoptosis. FASEB J. 2007;21(1):188-96.

34. Mukamel Z, Kimchi A. Death-associated protein 3 localizes to the mitochondria and is involved in the process of mitochondrial fragmentation during cell death. J Biol Chem. 2004;279(35):36732-8.

35. Xiao L, Xian H, Lee KY, Xiao B, Wang H, Yu F, et al. Death-associated Protein 3 Regulates Mitochondrial-encoded Protein Synthesis and Mitochondrial Dynamics. J Biol Chem. 2015;290(41):24961-74.

36. Calvo SE, Clauser KR, Mootha VK. MitoCarta2.0: an updated inventory of mammalian mitochondrial proteins. Nucleic acids research. 2016;44(D1):D12517.

37. Louwagie EJ, Larsen TD, Wachal AL, Baack ML. Placental lipid processing in response to a maternal high-fat diet and diabetes in rats. Pediatr Res. 2018;83(3):712-22.

38. Blagosklonny MV. TOR-centric view on insulin resistance and diabetic complications: perspective for endocrinologists and gerontologists. Cell death disease. 2013;4:e964.

39. Ratajczak MZ, Bartke A, Darzynkiewicz Z. Prolonged Growth Hormone/Insulin/Insulin-like Growth Factor Nutrient Response Signaling Pathway as a Silent Killer of Stem Cells and a Culprit in Aging. Stem cell reviews reports. 2017;13(4):443-53.

40. Schultze SM, Hemmings BA, Niessen M, Tschopp O. PI3K/AKT, MAPK and AMPK signalling: protein kinases in glucose homeostasis. Expert Rev Mol Med. 2012;14:e1.

41. Omar MA, Wang L, Clanachan AS. Cardioprotection by GSK-3 inhibition: role of enhanced glycogen synthesis and attenuation of calcium overload. Cardiovascular research. 2010;86(3):478-86.

42. Sakamaki J, Daitoku H, Kaneko Y, Hagiwara A, Ueno K, Fukamizu A. GSK3beta regulates gluconeogenic gene expression through HNF4alpha and FOXO1. J Recept Signal Transduct Res. 2012;32(2):96-101.

43. Lloyd-Jones DM, Leip EP, Larson MG, D'Agostino RB, Beiser A, Wilson PW, et al. Prediction of lifetime risk for cardiovascular disease by risk factor burden at 50 years of age. Circulation. 2006;113(6):791-8.

44. Ullmo S, Vial Y, Di Bernardo S, Roth-Kleiner M, Mivelaz Y, Sekarski N, et al. Pathologic ventricular hypertrophy in the offspring of diabetic mothers: a retrospective study. European heart journal. 2007;28(11):1319-25.

45. Freinkel N. Banting L. 1980. Of pregnancy and progeny. Diabetes. 1980;29(12):1023-35.

46. Lopaschuk GD, Ussher JR, Folmes CD, Jaswal JS, Stanley WC. Myocardial fatty acid metabolism in health and disease. Physiological reviews. 2010;90(1):207-58.

47. Kodde IF, van der Stok J, Smolenski RT, de Jong JW. Metabolic and genetic regulation of cardiac energy substrate preference. Comp Biochem Physiol A Mol Integr Physiol. 2007;146(1):26-39.

48. Goldberg IJ, Trent CM, Schulze PC. Lipid metabolism and toxicity in the heart. Cell Metabol. 2012;15(6):805-12.

49. Bertrand L, Horman S, Beauloye C, Vanoverschelde JL. Insulin signalling in the heart. Cardiovascular research. 2008;79(2):238-48.

50. Ernande L, Derumeaux G. Diabetic cardiomyopathy: myth or reality? Archives of cardiovascular diseases. 2012;105(4):218-25.

51. Louwagie EJ, Larsen TD, Wachal AL, Baack ML. Placental lipid processing in response to a maternal high-fat diet and diabetes in rats. Pediatric research. 2017.

52. Lehman JJ, Barger PM, Kovacs A, Saffitz JE, Medeiros DM, Kelly DP. Peroxisome proliferator-activated receptor gamma coactivator-1 promotes cardiac mitochondrial biogenesis. J Clin Investig. 2000;106(7):847-56.

53. Gilde AJ, Van Bilsen M. Peroxisome proliferator-activated receptors (PPARS): regulators of gene expression in heart and skeletal muscle. Acta Physiol Scand. 2003;178(4):425-34.

54. Ashrafian H, Frenneaux MP, Opie LH. Metabolic mechanisms in heart failure. Circulation. 2007;116(4):434-48.

55. Taegtmeyer H, Young ME, Lopaschuk GD, Abel ED, Brunengraber H, Darley-Usmar V, et al. Assessing Cardiac Metabolism: A Scientific Statement From the American Heart Association. Circulation research. 2016;118(10):1659-701.

56. Kumar N, Afeyan R, Sheppard S, Harms B, Lauffenburger DA. Quantitative analysis of Akt phosphorylation and activity in response to EGF and insulin treatment. Biochem Biophys Res Commun. 2007;354(1):14-20.

57. Mdaki KS, Larsen TD, Weaver LJ, Baack ML. Age related bioenergetics profiles in isolated rat cardiomyocytes using extracellular flux analyses. PloS one. 2016;11(2):e0149002. 
58. Lopaschuk GD, Jaswal JS. Energy metabolic phenotype of the cardiomyocyte during development, differentiation, and postnatal maturation. J Cardiovasc Pharmacol. 2010;56(2):130-40.

59. Weber HS, Copel JA, Reece EA, Green J, Kleinman CS. Cardiac growth in fetuses of diabetic mothers with good metabolic control. J Pediatr. 1991;118(1):103-7.

60. Zablah JE, Gruber D, Stoffels G, Cabezas EG, Hayes DA. Subclinical Decrease in Myocardial Function in Asymptomatic Infants of Diabetic Mothers: A Tissue Doppler Study. Pediatr Cardiol. 2017;38(4):801-6.

61. Jennings RE, Berry AA, Strutt JP, Gerrard DT, Hanley NA. Human pancreas development. Development. 2015;142(18):3126-37.

62. Lee KK, Raja EA, Lee AJ, Bhattacharya S, Bhattacharya S, Norman JE, et al. Maternal Obesity During Pregnancy Associates With Premature Mortality and Major Cardiovascular Events in Later Life. Hypertension (Dallas, Tex: 1979). 2015;66(5):938 - 44.

63. Reynolds RM, Allan KM, Raja EA, Bhattacharya S, McNeill G, Hannaford PC, et al. Maternal obesity during pregnancy and premature mortality from cardiovascular event in adult offspring: follow-up of 1323275 person years. BMJ. 2013;347:f4539.

64. Perestrelo T, Correia M, Ramalho-Santos J, Wirtz D. Metabolic and Mechanical Cues Regulating Pluripotent Stem Cell Fate. Trends in cell biology. 2018;28(12):1014-29.

65. Mitra K. Mitochondrial fission-fusion as an emerging key regulator of cell proliferation and differentiation. BioEssays: news and reviews in molecular. cellular developmental biology. 2013;35(11):955-64.

66. Murphy E, Ardehali H, Balaban RS, DiLisa F, Dorn GW 2nd, Kitsis RN, et al. Mitochondrial Function, Biology, and Role in Disease: A Scientific Statement From the American Heart Association. Circulation research. 2016.

67. Dorn GW 2nd, Vega RB, Kelly DP. Mitochondrial biogenesis and dynamics in the developing and diseased heart. Genes Dev. 2015;29(19):1981-91.

68. Hoppel CL, Tandler B, Fujioka H, Riva A. Dynamic organization of mitochondria in human heart and in myocardial disease. Int J Biochem Cell Biol. 2009;41(10):1949-56.

69. Hollander JM, Thapa D, Shepherd DL. Physiological and structural differences in spatially distinct subpopulations of cardiac mitochondria: influence of cardiac pathologies. American journal of physiology Heart circulatory physiology. 2014;307(1):H1-14.

70. Austin S, St-Pierre J. PGC1alpha and mitochondrial metabolism-emerging concepts and relevance in ageing and neurodegenerative disorders. Journal of cell science. 2012;125(Pt 21):4963-71.

71. Rius-Perez S, Torres-Cuevas I, Millan I, Ortega AL, Perez S. PGC-1alpha, Inflammation, and Oxidative Stress: An Integrative View in Metabolism. Oxidative Med Cell Longev. 2020;2020:1452696.

72. De Silva D, Tu YT, Amunts A, Fontanesi F, Barrientos A. Mitochondrial ribosome assembly in health and disease. Cell cycle (Georgetown Tex). 2015;14(14):2226-50.

73. Brown A, Rathore S, Kimanius D, Aibara S, Bai XC, Rorbach J, et al. Structures of the human mitochondrial ribosome in native states of assembly. Nat Struct Mol Biol. 2017;24(10):866-9.

74. Yagi M, Uchiumi T, Takazaki S, Okuno B, Nomura M, Yoshida S, et al. p32/gC1qR is indispensable for fetal development and mitochondrial translation: importance of its RNA-binding ability. Nucleic acids research. 2012;40(19):9717-37.

75. Jia L, Kaur J, Stuart RA. Mapping of the Saccharomyces cerevisiae Oxa1-mitochondrial ribosome interface and identification of MrpL40, a ribosomal protein in close proximity to Oxa1 and critical for oxidative phosphorylation complex assembly. Eukaryot Cell. 2009;8(11):1792-802.

76. Hu M, Crawford SA, Henstridge DC, Ng IH, Boey EJ, Xu Y, et al. p32 protein levels are integral to mitochondrial and endoplasmic reticulum morphology, cell metabolism and survival. Biochem J. 2013;453(3):381-91.

77. Allen JW. Cytochrome c biogenesis in mitochondria-Systems III and V. FEBS J. 2011;278(22):4198-216.

78. San Francisco B, Bretsnyder EC, Kranz RG. Human mitochondrial holocytochrome c synthase's heme binding, maturation determinants, and complex formation with cytochrome c. Proc Natl Acad Sci U S A. 2013;110(9):E788-97.

79. Dzeja P, Terzic A. Adenylate kinase and AMP signaling networks: metabolic monitoring, signal communication and body energy sensing. Int J Mol Sci. 2009;10(4):1729-72.

\section{Figures}


A
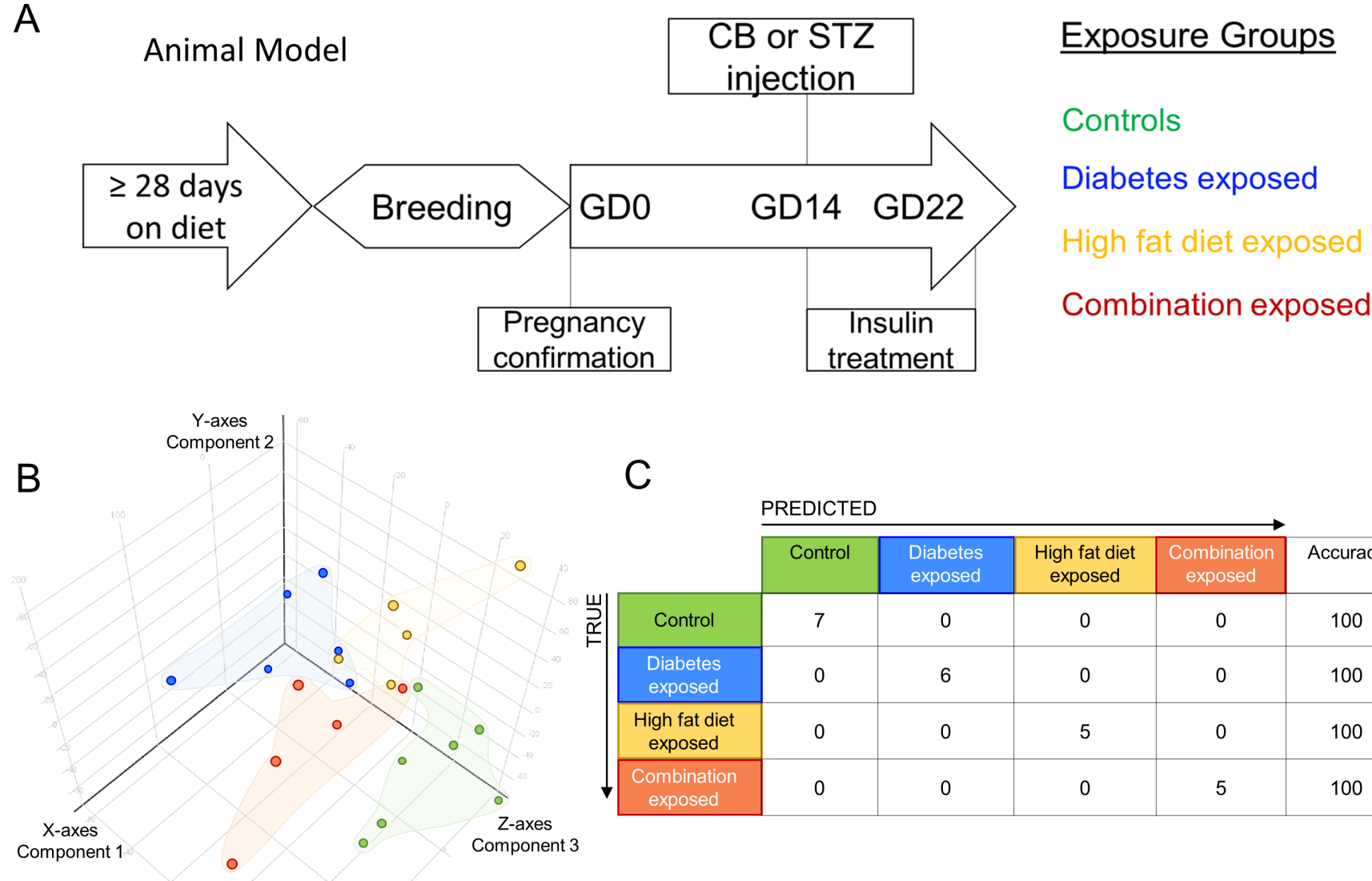

\begin{tabular}{|l|c|c|c|c|c|}
\multicolumn{1}{c}{ PREDICTED } \\
\cline { 2 - 6 } & Control & $\begin{array}{c}\text { Diabetes } \\
\text { exposed }\end{array}$ & $\begin{array}{c}\text { High fat diet } \\
\text { exposed }\end{array}$ & $\begin{array}{c}\text { Combination } \\
\text { exposed }\end{array}$ & Accuracy \\
\hline Control & 7 & 0 & 0 & 0 & 100 \\
\hline $\begin{array}{c}\text { Diabetes } \\
\text { exposed }\end{array}$ & 0 & 6 & 0 & 0 & 100 \\
\hline $\begin{array}{c}\text { High fat diet } \\
\text { exposed }\end{array}$ & 0 & 0 & 5 & 0 & 100 \\
\hline $\begin{array}{c}\text { Combination } \\
\text { exposed }\end{array}$ & 0 & 0 & 0 & 5 & 100 \\
\hline
\end{tabular}

\section{Figure 1}

Maternal diabetes and maternal high fat diet impart distinct cardiac transcriptome signatures in newborn rat offspring. A) Schematic showing experimental model of exposed groups. Female rats had at least 28 days of either control or high-fat diet prior to breeding. Female diet continued throughout pregnancy. At gestational day 14 a single injection of citrate buffer (CB) or streptozotocin (STZ) was delivered to a subset of females with high-fat or control diet. At GD22 newborns were delivered and hearts were extracted for study from four exposed groups: controls (green), diabetes exposed (blue), high fat diet exposed (yellow), and combination exposed (orange). B) Principal components analysis (PCA) plot depicting distinct transcriptome signature among all the exposed groups (component $1=25.1 \%$, component $2=8.9 \%$, component $3=4.4 \%$ ). C) Class prediction model used individual sample expression signatures to demonstrate with $100 \%$ accuracy the identification of each sample to their respective exposure group. 
A
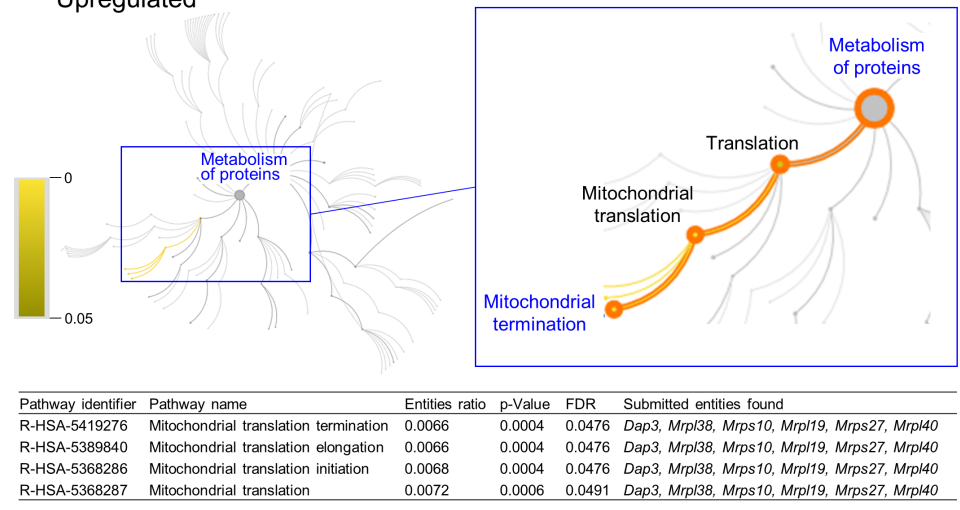

B

B Downregulated

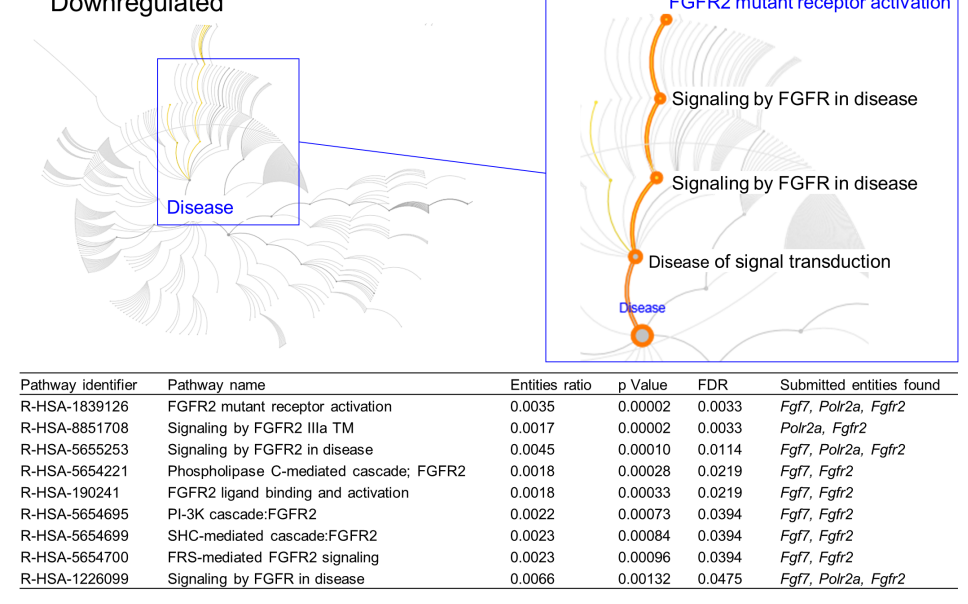

\section{Figure 2}

Functional pathways prioritized in differentially expressed genes from combination exposed group. Functional annotation and pathways analysis was done using Reactome pathway database in down and upregulated differentially expressed genes (DEGs) of combination exposed group when compared to control. A) Analysis of the upregulated DEGs elicited four mitochondrial translation-related pathways, associated with Dap3, Mrpl38, Mrps10, Mrpl19, Mrps27, Mrpl40.

B) Downregulated list revealed nine functional pathways directly associated with fibroblast growth factor receptor 2 (Fgfr2). 
A

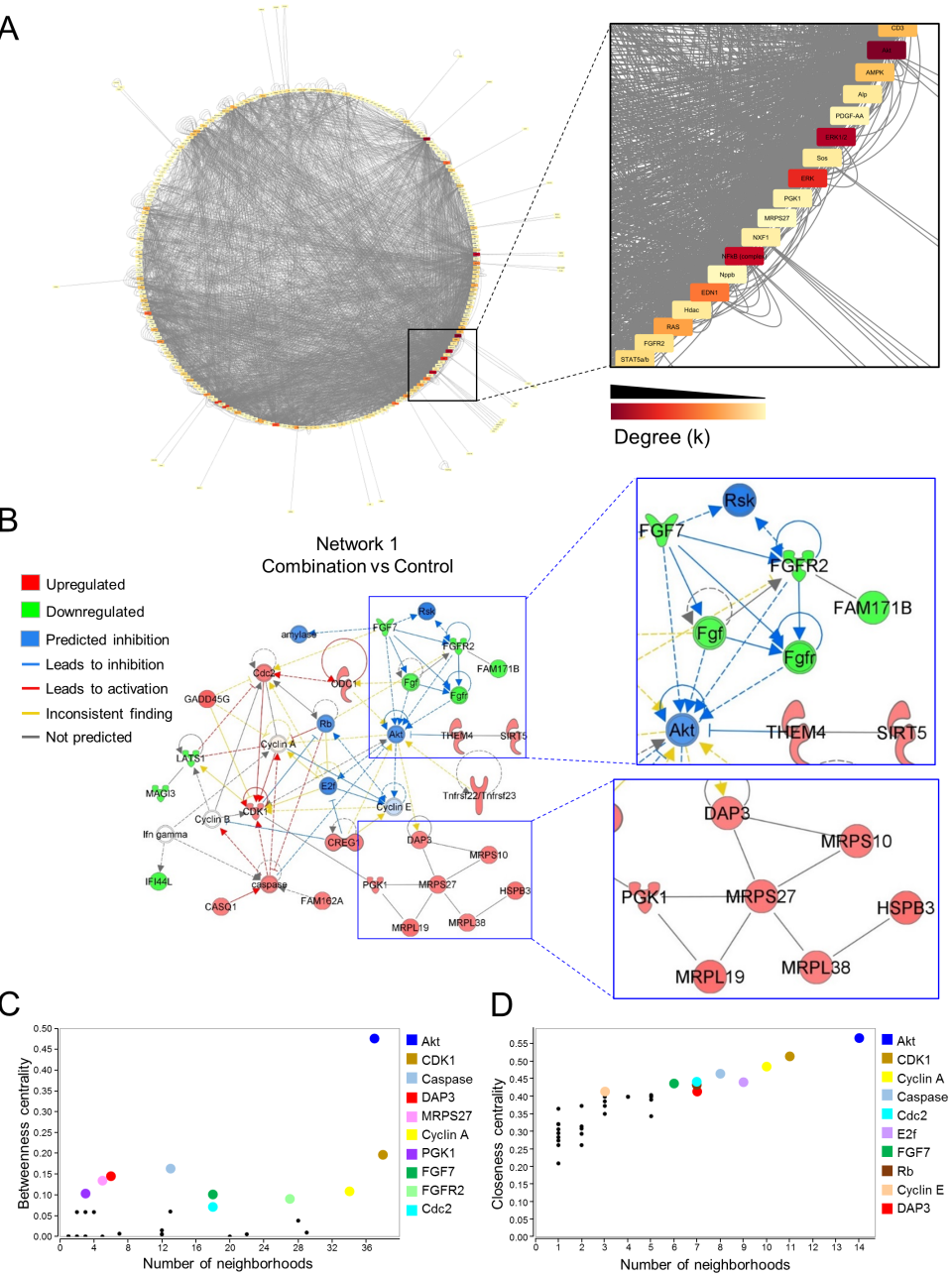

\section{Figure 3}

Akt is a critical hub within the network of transcriptome remodeling that characterizes hearts of combination exposed offspring. Interactions of merged functional networks, constructed in IPA from statistically significant genes in combination exposed group, were uploaded into Cytoscape for network analysis. A) Circular network layout depicting node interactions and colored by degree. Magnification of region with highest edge density (dark grey) showing Akt as the network node with the highest degree, followed by Erk1/2, Erk, NfkB (complex), Edn1, Ras, Ampk, Cd3, Alp, Fgfr2, Stat5a/b, Hdac, Pdgf-AA, Sos, Pgk1, Nxf1, Nppb and Mrps27 (highest to lowest degree respectively). B) IPA Molecule Predictor Analysis of the highest priority network (Network 1, Score 39, top functions: cell cycle, protein synthesis, hair and skin development/function) predicted inhibition (blue) of Akt in the combination exposed newborn heart by net expression of differentially expressed genes. Right: Magnification of Fgfr2 (downregulated in green) and Mrps27 (upregulated in red) hubs and immediate subnetwork neighborhood. C, D) Graph theory analysis of Network 1 (Combination vs control) revealed Akt as a network hub with the highest betweenness and closeness centrality metrics, indicative of its importance to overall network structure and communication. Among the top 10 nodes prioritized include: Cdk1, Caspase, Dap3, Mrps27, Cyclin A, Pgk1, Fgf7, Fgfr2, Cdc2, E2f and Rb. IPA = Ingenuity Pathways Analysis, Degree (k) = neighborhood connectivity distribution. 


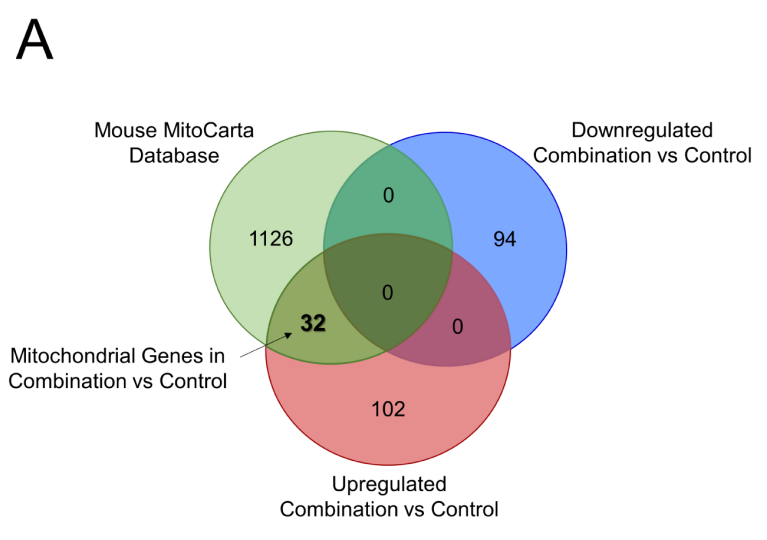

B

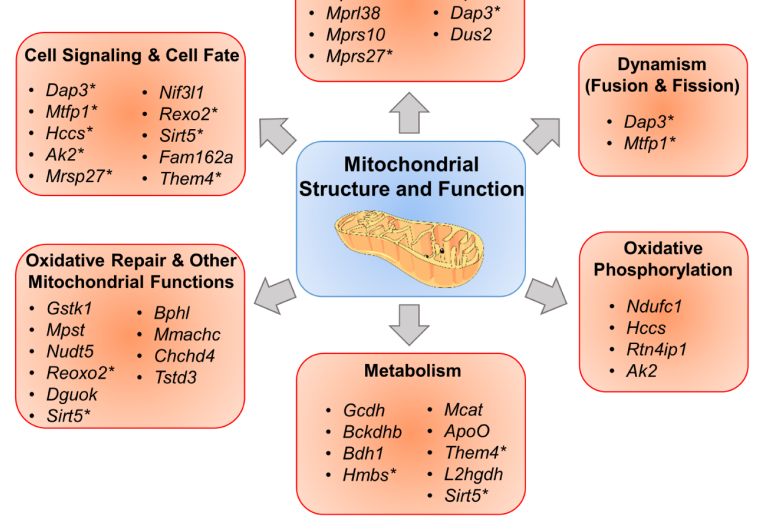

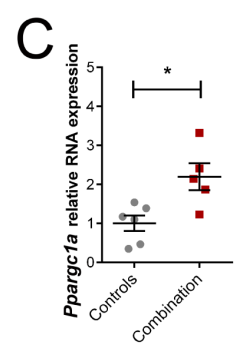
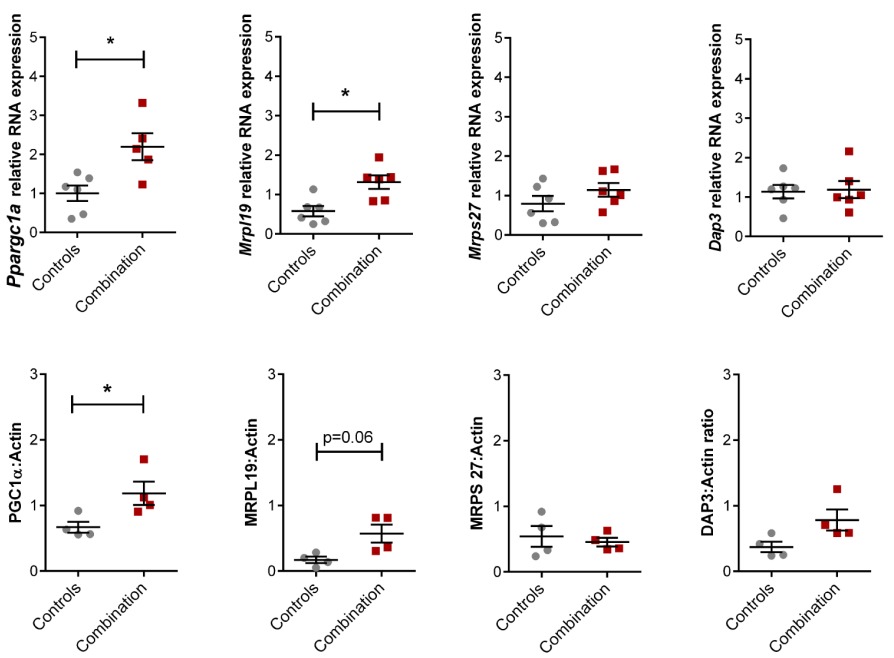

$\mathrm{D}$
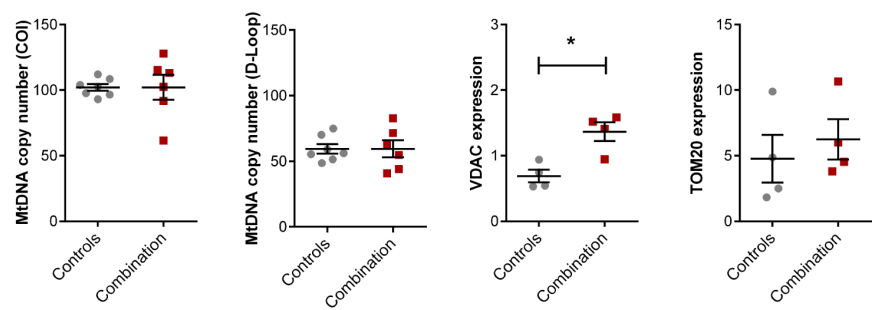

\section{Figure 4}

Combination exposed offspring hearts enriches discrete classes of mitochondrial genes. Genes that were duplicated or non-annotated were excluded, resulting in 228 differentially expressed genes (DEG) with 94 down and 134 upregulated in combination exposed hearts when compared to controls. A) The 228 DEGs were plotted in a Venn diagram against 1158 mitochondrial associated genes (MitoCarta Database, Mouse) and depicted 32 upregulated mitochondrial genes (Additional File 3: Table S7) with none downregulated in the combination exposed offspring hearts. B) Mitochondrial functions associated with the 32 upregulated mitochondrial genes were: cell signaling and cell fate, mitochondrial biogenesis, dynamism, oxidative phosphorylation, metabolism, oxidative repair and other mitochondrial functions. *Genes with multiple functions; shown in more than one category. C) RNA expression of Ppargc1a and Mrpl19 were significantly upregulated with fold change ( $F C)$ of 2.24 and 2.28 respectively $(p<0.05)$ in combination exposed newborn hearts (red squares) compared to controls (gray circles; $n=6$ /group). We showed no significant change in RNA expression of Mrps27 $(F C=1.09, p=0.6)$ or $D a p 3(F C=1.10, p=0.75)$. Consistent with the RNA expression, protein expression of PGC1a was significantly increased in newborn hearts exposed to diabetes and HF diet when compared to controls ( $n=4$ /group, $p<0.05$ ). We observed a non-significant upregulation trend for MRPL19 and DAP3 protein expressions with no change in MRPS27. D) Relative mtDNA copy number was not different by mtDNA expression of either cytochrome-c oxidase I (COI) or mitochondrial control region (Dloop). However, combination exposed hearts had higher protein expression of Voltage dependent anion channel 1 (VDAC) and a trend towards higher Translocase of outer mitochondrial membrane 20 (TOM20). 
A
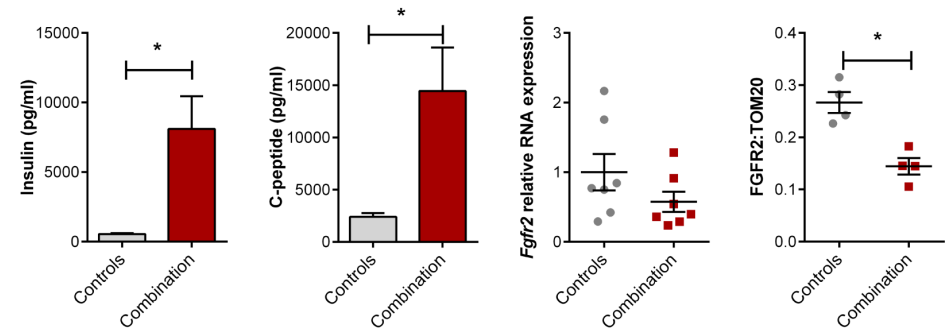

B
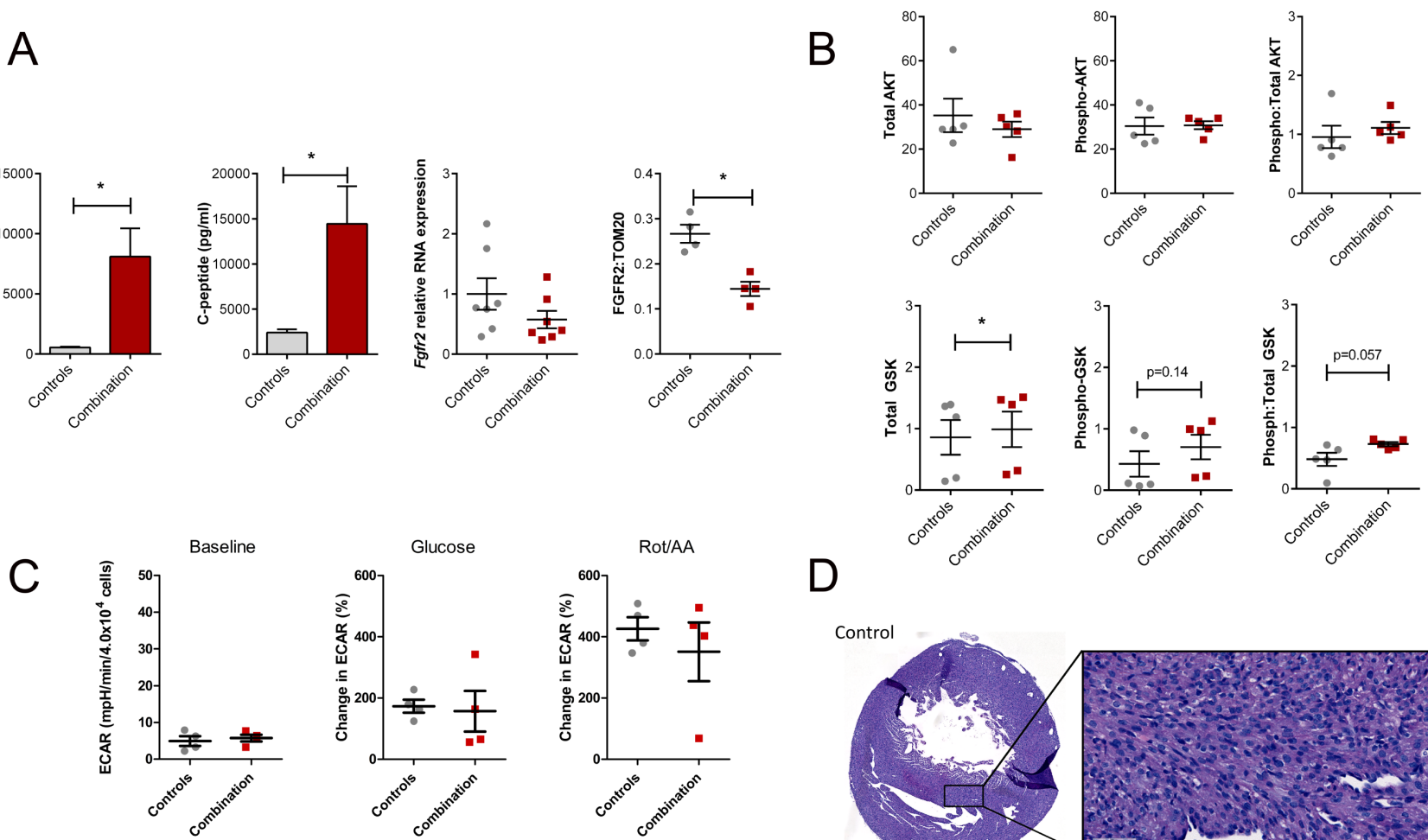

D

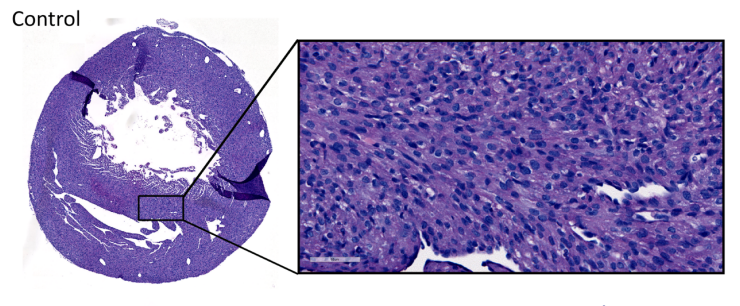

Combination Exposed

PAS

Hematoxylin
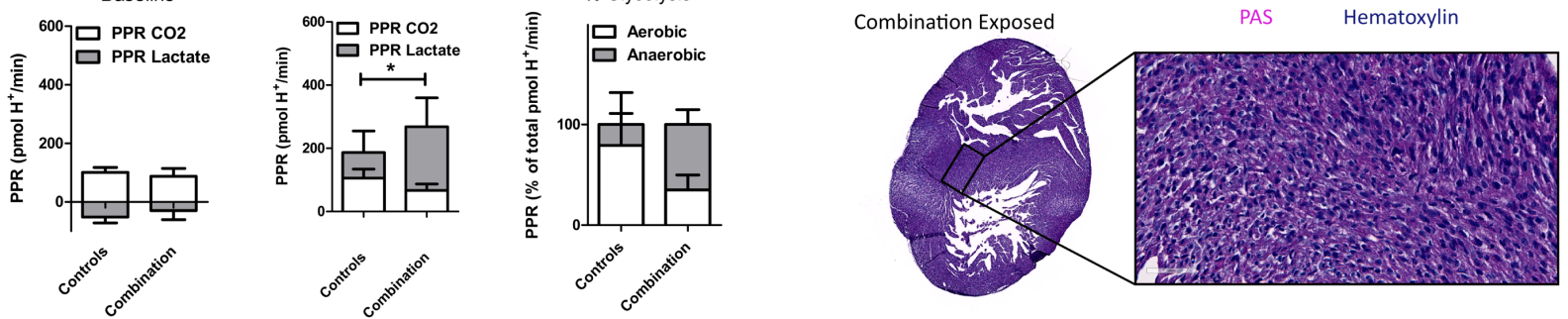

\section{Figure 5}

Evidence of insulin resistance in diabetes and high fat diet exposed offspring hearts. Chronic exposure to insulin and other growth hormones can cause insulin resistance through downregulation of growth factor receptors and impaired downstream activation of the PI3K/AKT pathway which shifts metabolism from glycolysis to gluconeogenesis/glycogen accumulation. A) Combination exposed newborn offspring ( $n=10 /$ group) had significantly higher circulating insulin and c-peptide levels. Consistent with transcriptome analyses, combination exposed newborn male hearts, had a trend towards lower RNA expression relative to B2m ( $p=0.13, n=7 /$ group) and lower protein expression ( $p<0.05, n=4 /$ group) of FGFR2. B) Total and phosphorylated AKT ( $n=5 /$ group) was not different, but GSK3 $\beta$ was higher ( $n=5 /$ group) and there was a trend towards more phosphorylated (active) and ratio of phosphorylated:total GSK3 $\beta$ ( $p=0.14$ and $p=0.057$ respectively) in combination exposed, male hearts. C) Primary isolated newborn rat cardiomyocytes (NRCM) from combination exposed male offspring had no significant difference in baseline extracellular acidification rate (ECAR), glucose or rotenone/antimycin (Rot/AA) stimulated glycolysis (glycolytic capacity) by XF analyses (top row). The proton production rate (PPR) was calculated to estimate aerobic (PPR from CO2) and anaerobic (PPR from lactate) glycolysis. At baseline, there was no difference, but aerobic glycolysis was significantly lower in combination exposed NRCM following glucose injection. Combination exposed NRCM had only $34 \%$ aerobic glycolysis vs. $79 \%$ aerobic glycolysis following glucose in controls. This suggests maternal diabetes and high fat diet exposure impairs aerobic glycolytic capacity. ${ }^{*} p<0.05, n=$ NRCM pooled from 3-4 pups/litter, 4 litters/group. E) Periodic Acid Schiff (PAS) staining demonstrates more glycogen deposition in combination exposed hearts, which suggests a chronic in utero switch from glucose utilization to storage occurred during development. 


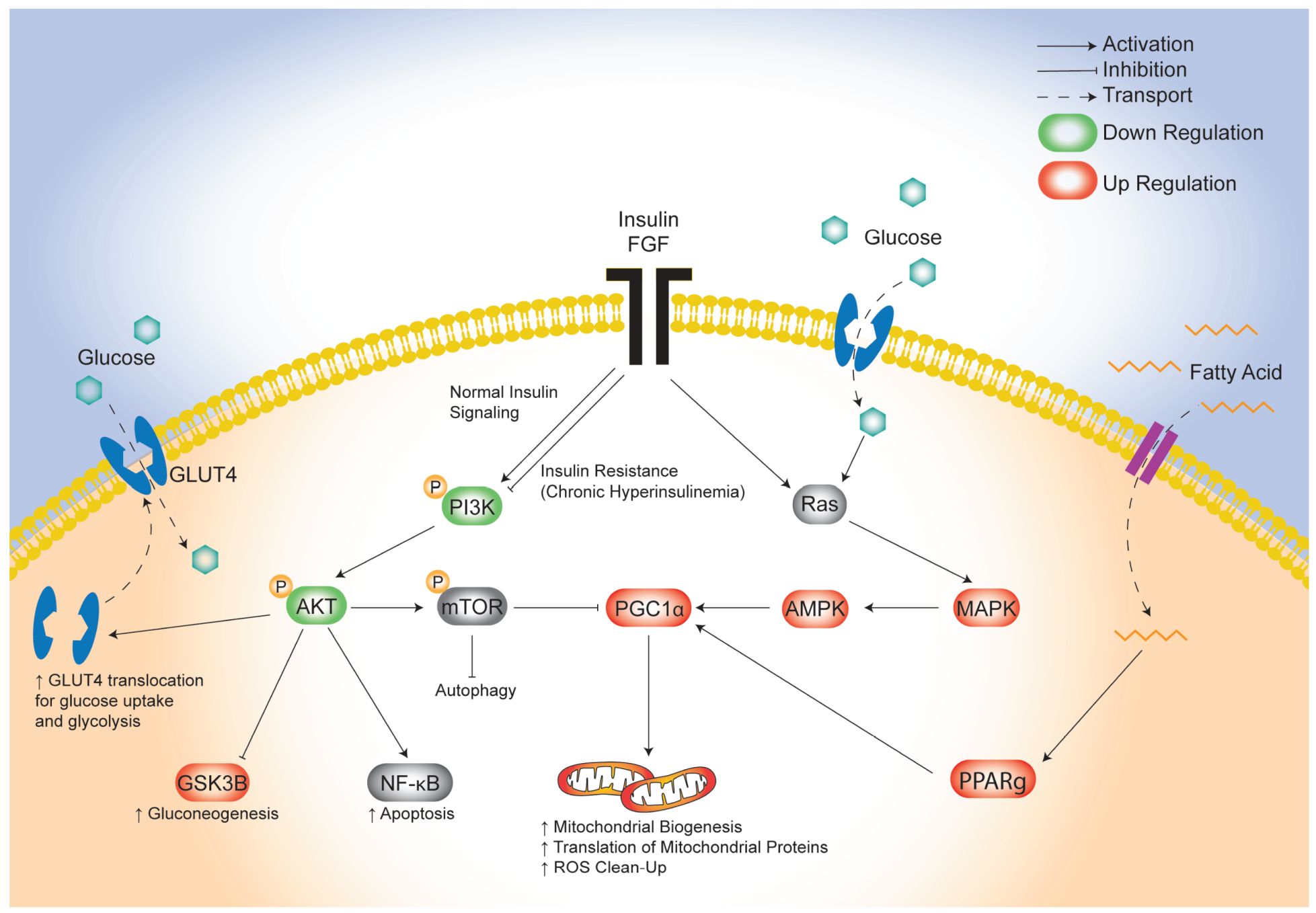

Figure 6

Proposed model of maternal combination exposed diet effects on cardiac network signaling in offspring. This illustrative schematic depicts the metabolic pathways in which the triad of maternal hyperglycemia, hyperlipidemia, and fetal hyperinsulinemia leads to an increased mitochondrial biogenesis, autophagy, and a shift from glycolysis to gluconeogenesis. This occurs via a downregulated PI3K/AKT pathway and increased PGC1a expression amongst uninhibited MAPK activity. AKT = protein kinase B; AMPK = 5' adenosine monophosphate-activated protein kinase; FGF = fibroblast growth factor; GLUT4 = glucose transporter type $4 ;$ GSK3 $=$ glycogen synthase kinase 3 beta; MAPK = mitogen-activated protein kinase; mTOR = mammalian target of rapamycin; NF$\mathrm{kB}=$ nuclear factor kappa-light-chain-enhancer of activated B cells; PGC1a = PPARG coactivator 1 alpha, PPARg = peroxisome proliferator activated receptor gamma; Ras = proto-oncogene protein p21.

\section{Supplementary Files}

This is a list of supplementary files associated with this preprint. Click to download.

- AdditionalFile3TableS7MitochondrialgenesCombvsControl.pdf

- AdditionalFile3TableS7MitochondrialgenesCombvsControl.pdf

- AdditionalFile1FigureS1VolcanoPlotsAllgroups.pdf

- AdditionalFile1FigureS1VolcanoPlotsAllgroups.pdf

- AdditionalFile2TablesS16DEGsAllgroups.pdf

- AdditionalFile2TablesS16DEGsAllgroups.pdf 